\title{
The mean electro-motive force and current helicity under the influence of rotation, magnetic field and shear.
}

\author{
Pipin V.V.*
}

October 20, 2018

Institute Solar-Terrestrial Physics, Irkutsk, Russia.

\begin{abstract}
The mean electromotive force (MEMF) in a rotating stratified magnetohydrodynamical turbulence is studied. Our study rests on the mean-field magnetohydrodynamics framework and $\tau$ approximation. We compute the effects of the large-scale magnetic fields (LSMF), global rotation and large-scale shear flow on the different parts of the MEMF (such as $\alpha$ - effect, turbulent diffusion, turbulent transport, etc.) in an explicit form. The influence of the helical magnetic fluctuations which stem from the small-scale dynamo is taken into account, as well. In the paper, we derive the equation governing the current helicity evolution. It is shown that the joint effect of the differential rotation and magnetic fluctuations in the stratified media can be responsible for the generation, maintenance and redistribution of the current helicity. The implication of the obtained results to astrophysical dynamos is considered as well.
\end{abstract}

\section{Introduction}

The mean-field magnetohydrodynamics presents one of the most powerful tools for exploring the nature of the large-scale magnetic activity in cosmic bodies (Moffatt, 1978; Parker, 1979; Krause and Rädler, 1980). It is widely believed that magnetic field generation there is governed by interplay between turbulent motions of electrically conductive fluids and global rotation. The growth and evolution of the large-scale magnetic fields (LSMF) in cosmic plasma strongly depends on the mean electromotive force, $\mathcal{E}=\langle\mathbf{u} \times \mathbf{b}\rangle$, which is given by the correlation between the fluctuating components of the velocity field of plasma, $\mathbf{u}$, and the fluctuating magnetic fields, $\mathbf{b}$.

The global rotation, stratification and the strong LSMF can substantially modify the structure and amplitude of the mean electromotive force (hereafter, MEMF) leading to the rich variety of the turbulence effects driving the evolution of the LSMF in cosmic bodies, e.g., the $\alpha$-effect (Roberts and Soward, 1975; Moffatt, 1978; Krause and Rädler, 1980; Parker, 1979; Rüdiger and Kichatinov, 1993), the rotationally-induced anisotropy of turbulent diffusion and effective drift of LSMF (Roberts and Soward, 1975; Krause and Rädler, 1980; Kichatinov et al., 1994), etc.. Generally speaking, the nonlinear effects of the small-scale Lorentz forces on the MEMF and LSMF evolution stem from two sources. One is driven by perturbations of the LSMF due to turbulent motions and another one is due to magnetic fluctuations, which are maintained by the small-scale dynamo action in a turbulent medium. The role of the small-scale dynamo in the LSMF evolution is still insufficiently understood. Numerous contributions to this subject can be found in the modern literature, e.g., (Moffatt, 1978; Frisch et al., 1975; Pouquet et al., 1975; Brandenburg and Subramanian, 2005). According to the mentioned studies the most important effect of the growing magnetic fluctuations on the LSMF evolution is caused by the helical part of magnetic fluctuations. The magnetic helicity conservation law, if applied to the mean-field magnetohydrodynamics, requires that the amount of helicity contained in the LSMF (controlled mostly by $\alpha$-effect) should be roughly

*pip@iszf.irk.ru 
the same and opposite in sign to its counterpart in the small scales, see (Kleeorin and Ruzmaikin, 1982). In this way the helical part of magnetic fluctuations, which is excited both due to shredding the LSMF by turbulent motions and due to small-scale dynamo, effectively saturates the generation of the LSMF by $\alpha$-effect (Vainshtein and Kitchatinov (1983); Brandenburg (2001); Field and Blackman (2002); Blackman and Brandenburg (2002)). Further discussions on this subject can be found in above cited papers. Their main lesson is that the construction of the realistic mean-field dynamo theory requires the evolution of the small scale magnetic (or current-) helicity to be taken into account.

Currently, there are two basic schemes for computing the MEMF of turbulent fields. One is the quasi-linear approximation (the same approximation is called the FOSA or SOCA in literature). A comprehensive discussion about its applicability and validity in astrophysics can be found at papers by Moffatt (1978); Parker (1979); Krause and Rädler (1980); Brandenburg and Subramanian (2005). This scheme remains one of the main tools of the mean-field magnetohydrodynamics. However, one of unfortunate problem of SOCA is that the contribution of the magnetic fluctuations (and the corresponding magnetic helicity) driven by the small-scale dynamo is hardly possible to include in the theory in self-consistent way. The third order closure scheme based on $\tau$-approximation (Orszag (1970); Vainshtein and Kitchatinov (1983); Rädler et al. (2003); Brandenburg and Subramanian (2005)) gives a chance to consider, roughly, the effects of the small-scale dynamo on the MEMF. Following (Brandenburg and Subramanian, 2005) (hereafter BS05), I will call it MTA (minimal tau approximation). Different kinds of this approximation are used in the literature, see (Vainshtein, 1983; Vainshtein and Kitchatinov, 1983; Rädler et al., 2003; Brandenburg and Subramanian, 2005; Rogachevskii and Kleeorin, 2003; Blackman and Field, 2002; Field and Blackman, 2002). In the paper we follow procedure described in BS05. Furthermore, the variant of tau approximation with a scale-independent relaxation time, $\tau$, is applied. For this reason, some results obtained in the paper can be different of those that are given elsewhere: (Rogachevskii and Kleeorin, 2003, 2004 b; ; Rädler et al., 2003).

The main purpose of this paper is to compute the MEMF via MTA taking into account the influence of the global rotation and LSMF on the turbulence. The stratification of the medium and the large-scale shear are taken into account as well. The influence of rotation, LSMF and uniform shear on the different parts of the MEMF (such as $\alpha$ - effect, turbulent diffusion, turbulent transport and etc.) is explicitly defined via factors describing the efficiency of rotational and LSMF feedback on the turbulent flows. The influence of rotation is measured by the Coriolis number, $\Omega^{*}=2 \Omega \tau_{c}$, where $\Omega$ is the solid body angular velocity and $\tau_{c}$ - the typical correlation time of turbulent flows. The influence of LSMF is measured by $\beta=\bar{B} /\left(u_{c} \sqrt{\mu \rho}\right)$, where $\bar{B}$ is the strength of the LSMF, $u_{c}$ is a typical rms velocity of turbulent flows and $\mu, \rho$ are the magnetic permeability and the density of the media, respectively. Following the basic approach developed in above cited papers we derive the equations governing the evolution of the current helicity both in rotating and in magnetized turbulent flows with imposed uniform shear.

The paper is structured as follows. In the next section we shortly outline the basic equations, assumptions and the computational scheme for derivation of the MEMF and the evolutionary equation for current helicity. Section 3 is devoted to the results of calculations of the MEMF for different situations (slow rotation, strong LSMF, vice versa and etc.). In section 4 we derive the evolutionary equation for current helicity. In section 5 we summarize the main results of the paper.

\section{Basic equations}

In the spirit of the mean-field magnetohydrodynamics, we split the physical quantities of the turbulent conducting fluid into the mean and randomly fluctuating part with the mean part defined as the ensemble average of the random fields. One assumes the validity of the Reynolds rules. The magnetic field $\mathbf{B}$ and velocity of motions $\mathbf{V}$ are decomposed as follows: $\mathbf{B}=\overline{\mathbf{B}}+\mathbf{b}$, $\mathbf{V}=\overline{\mathbf{V}}+\mathbf{u}$. Hereafter, everywhere, we use the small letters for the fluctuating part of the fields and capital letters with a bar above for the mean fields. The angle brackets are used for the ensemble 
average of products. Following the lines of two-scale approximation (Roberts and Soward, 1975; Krause and Rädler, 1980) we assume that the mean fields vary over the much larger scales (both in time and in space) than the fluctuating fields. The average effect of the MHD-turbulence on the LSMF evolution is described by the MEMF, $\mathcal{E}=\langle\mathbf{u} \times \mathbf{b}\rangle$. The governing equations for fluctuating magnetic field and velocity are written in a rotating coordinate system as follows

$$
\begin{aligned}
\frac{\partial \mathbf{b}}{\partial t} & =\nabla \times(\mathbf{u} \times \overline{\mathbf{B}}+\overline{\mathbf{V}} \times \mathbf{b})+\eta \nabla^{2} \mathbf{b}+\mathfrak{G}, \\
\frac{\partial m_{i}}{\partial t}+2(\boldsymbol{\Omega} \times \mathbf{m})_{i} & =-\nabla_{i}\left(p-\frac{2}{3}(\mathbf{G} \cdot \mathbf{m}) \nu+\frac{(\mathbf{b} \cdot \overline{\mathbf{B}})}{2 \mu}\right)+\nu \Delta m_{i}+\nu(\mathbf{G} \cdot \nabla) m_{i} \\
& +\frac{1}{\mu} \nabla_{j}\left(\bar{B}_{j} b_{i}+\bar{B}_{i} b_{j}\right)-\nabla_{j}\left(\bar{V}_{j} m_{i}+\bar{V}_{i} m_{j}\right)+f_{i}+\mathfrak{F}_{i},
\end{aligned}
$$

where $\mathfrak{G}, \mathfrak{F}$ are nonlinear contributions of fluctuating fields, $\mathbf{m}=\bar{\rho} \mathbf{u}, \mathbf{G}=\nabla \log \bar{\rho}$ is the density stratification scale of the media, $p$ - the fluctuating pressure, $\boldsymbol{\Omega}$ - the angular velocity responsible for the Coriolis force, $\overline{\mathbf{V}}$ - mean flow which is a weakly variable in space, $\mathbf{f}$ - the random force driving the turbulence.

To compute $\mathcal{E}$ it is convenient to write equations (11) and (2) in Fourier space:

$$
\begin{aligned}
\left(\frac{\partial}{\partial t}+\eta z^{2}\right) \hat{b}_{j} & =\mathrm{i} z_{l} \int\left[\widehat{m}_{j}(\mathbf{z}-\mathbf{q})\left(\frac{\hat{\bar{B}}_{l}}{\rho}\right)(\mathbf{q})-\widehat{m}_{l}(\mathbf{z}-\mathbf{q})\left(\frac{\hat{\bar{B}}_{j}}{\rho}\right)(\mathbf{q})\right] \mathrm{d} \mathbf{q} \\
& +\mathrm{i} z_{l} \int\left[\widehat{b}_{l}(\mathbf{z}-\mathbf{q}) \hat{\bar{V}}_{j}(\mathbf{q})-\widehat{b}_{j}(\mathbf{z}-\mathbf{q}) \hat{\bar{V}}_{l}(\mathbf{q})\right] \mathrm{d} \mathbf{q}+\widehat{\mathfrak{G}}_{j} \\
\left(\frac{\partial}{\partial t}+\nu z^{2}+\mathrm{i} \nu(\mathbf{G z})\right) \hat{m}_{i} & =\hat{f}_{i}+\hat{\mathfrak{F}}_{i}-2(\boldsymbol{\Omega} \hat{\mathbf{z}})(\hat{\mathbf{z}} \times \hat{\mathbf{m}})_{i} \\
& -\mathrm{i} \pi_{i f}(\mathbf{z}) z_{l} \int\left[\widehat{m}_{l}(\mathbf{z}-\mathbf{q}) \hat{\bar{V}}_{f}(\mathbf{q})+\widehat{m}_{f}(\mathbf{z}-\mathbf{q}) \hat{\bar{V}}_{l}(\mathbf{q})\right] \mathrm{d} \mathbf{q} \\
& +\frac{\mathrm{i}}{\mu} \pi_{i f}(\mathbf{z}) z_{l} \int\left[\widehat{b}_{l}(\mathbf{z}-\mathbf{q}) \hat{\bar{B}}_{f}(\mathbf{q})+\widehat{b}_{f}(\mathbf{z}-\mathbf{q}) \hat{\bar{B}}_{l}(q)\right] \mathrm{d} \mathbf{q},
\end{aligned}
$$

where the turbulent pressure was excluded from (2) by convolution with the projection tensor $\pi_{i j}(\mathbf{z})=\delta_{i j}-\hat{z}_{i} \hat{z}_{j}, \delta_{i j}$ is the Kronecker symbol and $\hat{\mathbf{z}}$ is a unit wave vector. The equations for the second-order moments which make contributions to the MEMF can be found from (3/4). As the preliminary step we write the equations for the second-order products of the fluctuating fields, and make the ensemble averaging of them,

$$
\begin{aligned}
\frac{\partial}{\partial t}\left\langle\hat{m}_{i}(\mathbf{z}) \hat{b}_{j}\left(\mathbf{z}^{\prime}\right)\right\rangle= & T h_{i j}^{\varkappa}\left(\mathbf{z}, \mathbf{z}^{\prime}\right)-\left(\eta z^{\prime 2}+\nu z^{2}+\mathrm{i} \nu(\mathbf{G} \mathbf{z})\right)\left\langle\hat{m}_{i}(\mathbf{z}) \hat{b}_{j}\left(\mathbf{z}^{\prime}\right)\right\rangle \\
& \mathrm{i} z_{l}^{\prime} \int\left[\left\langle\hat{m}_{i}(\mathbf{z}) \hat{m}_{j}\left(\mathbf{z}^{\prime}-\mathbf{q}\right)\right\rangle\left(\frac{\hat{B}_{l}}{\rho}\right)(\mathbf{q})-\right. \\
& \left.-\left\langle\hat{m}_{i}(\mathbf{z}) \hat{m}_{l}\left(\mathbf{z}^{\prime}-\mathbf{q}\right)\right\rangle\left(\frac{\hat{B}_{j}}{\rho}\right)(\mathbf{q})\right] \mathrm{d} \mathbf{q}-2(\boldsymbol{\Omega} \hat{\mathbf{z}}) \varepsilon_{i l n} \hat{z}_{l}\left\langle\hat{m}_{n}(\mathbf{z}) \hat{b}_{j}\left(\mathbf{z}^{\prime}\right)\right\rangle \\
+ & \mathrm{i} z_{l}^{\prime} \int\left[\left\langle\hat{m}_{i}(\mathbf{z}) \widehat{b}_{l}\left(\mathbf{z}^{\prime}-\mathbf{q}\right)\right\rangle \hat{\bar{V}}_{j}(\mathbf{q})-\left\langle\hat{m}_{i}(\mathbf{z}) \widehat{b}_{j}\left(\mathbf{z}^{\prime}-\mathbf{q}\right)\right\rangle \hat{\bar{V}}_{l}(\mathbf{q})\right] \mathrm{d} \mathbf{q} \\
& -\mathrm{i} \pi_{i f}(\mathbf{z}) z_{l} \int\left[\left\langle\widehat{m}_{l}(\mathbf{z}-\mathbf{q}) \hat{b}_{j}\left(\mathbf{z}^{\prime}\right)\right\rangle \hat{\bar{V}}_{f}(\mathbf{q})+\left\langle\widehat{m}_{f}(\mathbf{z}-\mathbf{q}) \hat{b}_{j}\left(\mathbf{z}^{\prime}\right)\right\rangle \hat{\bar{V}}_{l}(\mathbf{q})\right] \mathrm{d} \mathbf{q} \\
& +\frac{\mathrm{i}}{\mu} z_{l} \pi_{i f}(\mathbf{z}) \int\left[\left\langle\widehat{b}_{l}(\mathbf{z}-\mathbf{q}) \hat{b}_{j}\left(\mathbf{z}^{\prime}\right)\right\rangle \bar{B}_{f}(\mathbf{q})+\left\langle\widehat{b}_{f}(\mathbf{z}-\mathbf{q}) \hat{b}_{j}\left(\mathbf{z}^{\prime}\right)\right\rangle \bar{B}_{l}(q)\right] \mathrm{d} \mathbf{q},
\end{aligned}
$$




$$
\begin{aligned}
\frac{\partial}{\partial t}\left\langle\hat{m}_{i}(\mathbf{z}) \hat{m}_{j}\left(\mathbf{z}^{\prime}\right)\right\rangle= & -2(\boldsymbol{\Omega} \hat{\mathbf{z}}) \varepsilon_{i l n} \hat{z}_{l}\left\langle\hat{m}_{n}(\mathbf{z}) \hat{m}_{j}\left(\mathbf{z}^{\prime}\right)\right\rangle-2\left(\boldsymbol{\Omega} \hat{\mathbf{z}}^{\prime}\right) \varepsilon_{j l n} \hat{z}_{l}^{\prime}\left\langle\hat{m}_{i}(\mathbf{z}) \hat{m}_{n}\left(\mathbf{z}^{\prime}\right)\right\rangle \\
& -\mathrm{i} \pi_{i f}(\mathbf{z}) z_{l} \int\left[\left\langle\hat{m}_{l}(\mathbf{z}-\mathbf{q}) \hat{m}_{j}\left(\mathbf{z}^{\prime}\right)\right\rangle \hat{\bar{V}}_{f}(\mathbf{q})+\left\langle\hat{m}_{f}(\mathbf{z}-\mathbf{q}) \hat{m}_{j}\left(\mathbf{z}^{\prime}\right)\right\rangle \hat{\bar{V}}_{l}(\mathbf{q})\right] \mathrm{d} \mathbf{q} \\
& -\mathrm{i} \pi_{j f}\left(\mathbf{z}^{\prime}\right) z_{l}^{\prime} \int\left[\left\langle\hat{m}_{i}(\mathbf{z}) \hat{m}_{l}(\mathbf{z}-\mathbf{q})\right\rangle \hat{\bar{V}}_{f}(\mathbf{q})+\left\langle\hat{m}_{i}(\mathbf{z}) \hat{m}_{f}(\mathbf{z}-\mathbf{q})\right\rangle \hat{\bar{V}}_{l}(\mathbf{q})\right] \mathrm{d} \mathbf{q} \\
& +\frac{\mathrm{i}}{\mu} \pi_{i f}(\mathbf{z}) z_{l} \int\left[\left\langle\widehat{b}_{l}(\mathbf{z}-\mathbf{q}) \hat{m}_{j}\left(\mathbf{z}^{\prime}\right)\right\rangle \hat{\bar{B}}_{f}(\mathbf{q})+\left\langle\widehat{b}_{f}(\mathbf{z}-\mathbf{q}) \hat{m}_{j}\left(\mathbf{z}^{\prime}\right)\right\rangle \hat{\bar{B}}_{l}(q)\right] \mathrm{d} \mathbf{q} \\
& +\frac{\mathrm{i}}{\mu} \pi_{j f}\left(\mathbf{z}^{\prime}\right) z_{l}^{\prime} \int\left[\left\langle\hat{m}_{i}(\mathbf{z}) \widehat{b}_{l}(\mathbf{z}-\mathbf{q})\right\rangle \hat{\bar{B}}_{f}(\mathbf{q})+\left\langle\hat{m}_{i}(\mathbf{z}) \widehat{b}_{f}(\mathbf{z}-\mathbf{q})\right\rangle \hat{\bar{B}}_{l}(q)\right] \mathrm{d} \mathbf{q} \\
& +T h_{i j}^{v}\left(\mathbf{z}, \mathbf{z}^{\prime}\right)-\nu\left(z^{\prime 2}+z^{2}+i(\mathbf{G z})+i\left(\mathbf{G} \mathbf{z}^{\prime}\right)\right)\left\langle\hat{m}_{i}(\mathbf{z}) \hat{m}_{j}\left(\mathbf{z}^{\prime}\right)\right\rangle, \\
\frac{\partial}{\partial t}\left\langle\hat{b}_{i}(\mathbf{z}) \hat{b}_{j}\left(\mathbf{z}^{\prime}\right)\right\rangle= & T h_{i j}^{h}\left(\mathbf{z}, \mathbf{z}^{\prime}\right)-\left(\eta z^{\prime 2}+\eta z^{2}\right)\left\langle\hat{b}_{i}(\mathbf{z}) \hat{b}_{j}\left(\mathbf{z}^{\prime}\right)\right\rangle \\
& +\mathrm{i} z_{l}^{\prime} \int\left[\left\langle\hat{b}_{i}(\mathbf{z}) \hat{m}_{j}\left(\mathbf{z}^{\prime}-\mathbf{q}\right)\right\rangle\left(\frac{\hat{\bar{B}}_{l}}{\rho}\right)(\mathbf{q})-\left\langle\hat{b}_{i}(\mathbf{z}) \hat{m}_{l}\left(\mathbf{z}^{\prime}-\mathbf{q}\right)\right\rangle\left(\frac{\hat{B}_{j}}{\rho}\right)(\mathbf{q})\right] \mathrm{d} \mathbf{q} \\
& +\mathrm{i} z_{l} \int\left[\left\langle\hat{m}_{i}(\mathbf{z}-\mathbf{q}) \hat{b}_{j}\left(\mathbf{z}^{\prime}\right)\right\rangle\left(\frac{\hat{B}_{l}}{\rho}\right)(\mathbf{q})-\left\langle\hat{m}_{l}(\mathbf{z}-\mathbf{q}) \hat{b}_{j}\left(\mathbf{z}^{\prime}\right)\right\rangle\left(\frac{\hat{B}_{i}}{\rho}\right)(\mathbf{q})\right] \mathrm{d} \mathbf{q} \\
& +\mathrm{i} z_{l}^{\prime} \int\left[\left\langle\hat{b}_{i}(\mathbf{z}) \widehat{b}_{l}\left(\mathbf{z}^{\prime}-\mathbf{q}\right)\right\rangle \hat{\bar{V}}_{j}(\mathbf{q})-\left\langle\hat{b}_{i}(\mathbf{z}) \widehat{b}_{j}\left(\mathbf{z}^{\prime}-\mathbf{q}\right)\right\rangle \hat{\bar{V}}_{l}(\mathbf{q})\right] \mathrm{d} \mathbf{q} \\
& +\mathrm{i} z_{l} \int\left[\left\langle\hat{b}_{l}(\mathbf{z}-\mathbf{q}) \widehat{b}_{j}\left(\mathbf{z}^{\prime}\right)\right\rangle \hat{\bar{V}}_{i}(\mathbf{q})-\left\langle\hat{b}_{i}(\mathbf{z}-\mathbf{q}) \widehat{b}_{j}\left(\mathbf{z}^{\prime}\right)\right\rangle \hat{\bar{V}}_{l}(\mathbf{q})\right] \mathrm{d} \mathbf{q},
\end{aligned}
$$

where, the terms $T h_{i j}^{(\varkappa, v, h)}$ involve the third-order moments of fluctuating fields and second-order moments of them with the forcing term.

To proceed further, it is convenient to introduce some notations which are used in the literature. The double Fourier transformation of an ensemble average of two fluctuating quantities, say $f$ and $g$, taken at equal times and at the different positions $\mathbf{x}, \mathbf{x}^{\prime}$, is given by

$$
\left\langle f(\mathbf{x}) g\left(\mathbf{x}^{\prime}\right)\right\rangle=\iint\left\langle\hat{f}(\mathbf{z}) \hat{g}\left(\mathbf{z}^{\prime}\right)\right\rangle e^{\mathrm{i}\left(\mathbf{z} \cdot \mathbf{x}+\mathbf{z}^{\prime} \cdot \mathbf{x}^{\prime}\right)} \mathrm{d}^{3} \mathbf{z} \mathrm{d}^{3} \mathbf{z}^{\prime} .
$$

Let us define the "fast" spatial variable $\mathbf{r}$ by the relative difference of $\mathbf{x}, \mathbf{x}^{\prime}$ coordinates, $\mathbf{r}=\mathbf{x}-\mathbf{x}^{\prime}$. The "slow" spatial variable $\mathbf{R}$ is $\mathbf{R}=\left(\mathbf{x}+\mathbf{x}^{\prime}\right) / 2$. Then, eq. (8) can be written in the form

$$
\left\langle f(\mathbf{x}) g\left(\mathbf{x}^{\prime}\right)\right\rangle=\iint\left\langle\hat{f}\left(\mathbf{k}+\frac{1}{2} \mathbf{K}\right) \hat{g}\left(-\mathbf{k}+\frac{1}{2} \mathbf{K}\right)\right\rangle \mathrm{e}^{\mathrm{i}(\mathbf{K} \cdot \mathbf{R}+\mathbf{k} \cdot \mathbf{r})} \mathrm{d}^{3} \mathbf{K} \mathrm{d}^{3} \mathbf{k},
$$

where I have introduced two wave vectors: $\mathbf{k}=\left(\mathbf{z}-\mathbf{z}^{\prime}\right) / 2$ and $\mathbf{K}=\mathbf{z}+\mathbf{z}^{\prime}$. Following BS05, we define the correlation function of $\hat{\mathbf{f}}$ and $\hat{\mathbf{g}}$ obtained from (9) by integration with respect to $\mathbf{K}$,

$$
\Phi(\hat{f}, \hat{g}, \mathbf{k}, \mathbf{R})=\int\left\langle\hat{f}\left(\mathbf{k}+\frac{1}{2} \mathbf{K}\right) \hat{g}\left(-\mathbf{k}+\frac{1}{2} \mathbf{K}\right)\right\rangle \mathrm{e}^{\mathrm{i}(\mathbf{K} \cdot \mathbf{R})} \mathrm{d}^{3} \mathbf{K} .
$$

For further convenience we define the second order correlations of momentum density, magnetic fluctuations and the cross-correlations of momentum and magnetic fluctuations via

$$
\begin{aligned}
& \hat{v}_{i j}(\mathbf{k}, \mathbf{R})=\Phi\left(\hat{m}_{i}, \hat{m}_{j}, \mathbf{k}, \mathbf{R}\right), \bar{\rho}^{2}\left\langle u^{2}\right\rangle(\mathbf{R})=\int \hat{v}_{i i}(\mathbf{k}, \mathbf{R}) \mathrm{d}^{3} \mathbf{k}, \\
& \hat{h}_{i j}(\mathbf{k}, \mathbf{R})=\Phi\left(\hat{b}_{i}, \hat{b}_{j}, \mathbf{k}, \mathbf{R}\right),\left\langle b^{2}\right\rangle(\mathbf{R})=\int \hat{h}_{i i}(\mathbf{k}, \mathbf{R}) \mathrm{d}^{3} \mathbf{k}, \\
& \hat{\varkappa}_{i j}(\mathbf{k}, \mathbf{R})=\Phi\left(\hat{m}_{i}, \hat{b}_{j}, \mathbf{k}, \mathbf{R}\right), \bar{\rho} \mathcal{E}_{i}(\mathbf{R})=\varepsilon_{i j k} \int \hat{\varkappa}_{j k}(\mathbf{k}, \mathbf{R}) \mathrm{d}^{3} \mathbf{k} .
\end{aligned}
$$


Let us now return to equations (5), (6) and (7). As the first step, we approximate the $T h_{i j}^{(\varkappa, v, h)}$ terms by the corresponding $\tau$ relaxation terms of the second-order contributions,

$$
\begin{aligned}
T h_{i j}^{(\varkappa)} & \rightarrow-\left\langle\hat{m}_{i}(\mathbf{z}) \hat{b}_{j}\left(\mathbf{z}^{\prime}\right)\right\rangle / \tau_{c}, \\
T h_{i j}^{(v)} & \rightarrow-\frac{\left\langle\hat{m}_{i}(\mathbf{z}) \hat{m}_{j}\left(\mathbf{z}^{\prime}\right)\right\rangle-\left\langle\hat{m}_{i}(\mathbf{z}) \hat{m}_{j}\left(\mathbf{z}^{\prime}\right)\right\rangle^{(0)}}{\tau_{c}}, \\
T h_{i j}^{(h)} & \rightarrow-\frac{\left\langle\hat{b}_{i}(\mathbf{z}) \hat{b}_{j}\left(\mathbf{z}^{\prime}\right)\right\rangle-\left\langle\hat{b}_{i}(\mathbf{z}) \hat{b}_{j}\left(\mathbf{z}^{\prime}\right)\right\rangle^{(0)}}{\tau_{c}},
\end{aligned}
$$

where the superscript ${ }^{(0)}$ denotes the moments of the background turbulence. Here, $\tau_{c}$ is independent on $\mathbf{k}$ and it is independent on the mean fields as well. Furthermore, for the sake of simplicity, we restrict ourselves to the high Reynolds numbers limit and discard the microscopic diffusion terms. As the next step we make the Taylor expansion with respect to the "slow" variables and take the Fourier transformation, (10), about them. The details of this procedure can be found in BS05. In result, we obtain equations for the second order correlations of momentum density, magnetic fluctuations and the cross-correlations of momentum and magnetic fluctuations,

$$
\begin{aligned}
& \frac{\partial \hat{\varkappa}_{i j}}{\partial t}=-\mathrm{i}(\overline{\mathbf{B}} \mathbf{k})\left(\frac{\hat{v}_{i j}}{\rho}-\frac{\hat{h}_{i j}}{\mu}\right)+\frac{(\overline{\mathbf{B}} \nabla)}{2}\left(\frac{\hat{v}_{i j}}{\rho}+\frac{\hat{h}_{i j}}{\mu}\right)+\frac{(\overline{\mathbf{B}} \mathbf{k})}{2 \rho} G_{s} \frac{\partial \hat{v}_{i j}}{\partial k^{s}}-\frac{(\mathbf{G} \overline{\mathbf{B}})}{2 \rho} \hat{v}_{i j} \\
& +\frac{1}{\rho} G_{l} \hat{v}_{i l} B_{j}+\frac{\hat{h}_{l j} \bar{B}_{i, l}}{\mu}-\frac{\hat{v}_{i l} \bar{B}_{j, l}}{\rho}-\frac{k_{l} \bar{B}_{l, f}}{2} \frac{\partial}{\partial k_{f}}\left[\frac{\hat{v}_{i j}}{\rho}+\frac{\hat{h}_{i j}}{\mu}\right]-\frac{2}{\mu} \hat{k}_{i} \hat{k}_{f} \bar{B}_{f, l} \hat{h}_{l j} \\
& +\bar{V}_{j, l} \hat{\varkappa}_{i l}-\bar{V}_{i, l} \hat{\varkappa}_{l j}+2 \hat{k}_{i} \hat{k}_{f} \hat{\varkappa}_{l j} \bar{V}_{f, l}+k_{l} \bar{V}_{f, l} \frac{\partial \hat{\varkappa}_{i j}}{\partial k_{f}}-\frac{\hat{\varkappa}_{i j}}{\tau_{c}}-2(\Omega \hat{k}) \hat{k}_{p} \varepsilon_{i p l} \hat{\varkappa}_{l j} \\
& -2 \frac{\mathrm{i}}{k}(\Omega \hat{k}) \hat{k}_{p} \varepsilon_{i p l}(\hat{k} \nabla) \hat{\varkappa}_{l j}+\frac{\mathrm{i}}{k} \varepsilon_{i p l}\left((\Omega \hat{k}) \nabla_{p} \hat{\varkappa}_{l j}+\hat{k}_{p}(\Omega \nabla) \hat{\varkappa}_{l j}\right), \\
& \frac{\partial \hat{v}_{i j}}{\partial t}=-2(\Omega \hat{k}) \hat{k}_{p}\left(\varepsilon_{i p l} \hat{v}_{l j}+\varepsilon_{j p l} \hat{v}_{i l}\right)-\frac{\hat{v}_{i j}-\hat{v}_{i j}^{(0)}}{\tau_{c}}-\hat{v}_{l j} \bar{V}_{i, l}-\hat{v}_{i l} \bar{V}_{j, l} \\
& +2 \hat{k}_{f} \bar{V}_{f, l}\left(\hat{k}_{i} \hat{v}_{l j}+\hat{k}_{j} \hat{v}_{i l}\right)+k_{l} \bar{V}_{f, l} \frac{\partial \hat{v}_{i j}}{\partial k_{f}}-\mathrm{i}(\overline{\mathbf{B}} \mathbf{k})\left(\hat{\varkappa}_{i j}-\hat{\varkappa}_{j i}^{*}\right) \\
& +\frac{1}{2} \bar{B}_{l}\left(\hat{\varkappa}_{i j, l}+\hat{\varkappa}_{j i, l}^{*}\right)+\bar{B}_{i, l} \hat{\varkappa}_{j l}^{*}+\bar{B}_{j, l} \hat{\varkappa}_{i, l}-2 \hat{k}_{f} \bar{B}_{f, l}\left(\hat{k}_{i} \hat{\varkappa}_{j l}^{*}+\hat{k}_{j} \hat{\varkappa}_{i, l}\right) \\
& \text { - } \frac{\bar{B}_{l, f}}{2} k_{l} \frac{\partial}{\partial k_{f}}\left(\hat{\varkappa}_{i j}+\hat{\varkappa}_{j i}^{*}\right)+\frac{\mathrm{i}}{k} \varepsilon_{i p l}\left[\hat{k}_{p}((\Omega \nabla)-2(\Omega \hat{k})(\hat{\mathbf{k}} \nabla))\right. \\
& \left.+(\Omega \hat{k}) \nabla_{p}\right]\left(\varepsilon_{i p l} \hat{v}_{l j}-\varepsilon_{j p l} \hat{v}_{i l}\right) \text {, } \\
& \frac{\partial \hat{h}_{i j}}{\partial t}=-\frac{\hat{h}_{i j}-\hat{h}_{i j}^{(0)}}{\tau_{c}}+\hat{h}_{i l} \bar{V}_{j, l}+\hat{h}_{l j} \bar{V}_{i, l}+k_{l} \bar{V}_{f, l} \frac{\partial \hat{h}_{i j}}{\partial k_{f}}+\frac{\mathrm{i}(\overline{\mathbf{B}} \mathbf{k})}{\rho}\left(\hat{\varkappa}_{i j}-\hat{\varkappa}_{j i}^{*}\right) \\
& +\left\{\frac{(\bar{B} \nabla)}{2 \rho}-\frac{(\overline{\mathbf{B}} \mathbf{G})}{2 \rho}\right\}\left(\hat{\varkappa}_{i j}+\hat{\varkappa}_{j i}^{*}\right)-\left(\frac{\bar{B}_{j}}{\rho}\right)_{, l} \hat{\varkappa}_{l i}^{*}-\left(\frac{\bar{B}_{i}}{\rho}\right)_{, l} \hat{\varkappa}_{l j} \\
& -\frac{1}{2}\left(\frac{\bar{B}_{l}}{\rho}\right)_{, f} k_{l} \frac{\partial\left(\hat{\varkappa}_{i j}+\hat{\varkappa}_{j i}^{*}\right)}{\partial k_{f}}
\end{aligned}
$$

where $\hat{\varkappa}_{j i}^{*}=\Phi\left(\hat{b}_{j}, \hat{m}_{i}, \mathbf{k}, \mathbf{R}\right), \hat{\mathbf{k}}$ is the unit wave vector, the indexes behind the comma stand for the spatial derivatives. Equations (17[18]19) are in agreement with those considered in the paper by Rogachevskii and Kleeorin (2004a). 
To solve (17/18[19) we neglect the time derivatives at the left hand side of equations and apply the perturbation method. The mean field inhomogeneities and stratification scales of turbulence are considered as small. We shall not reproduce explicitly the rather bulky derivations which are explained elsewhere: Rogachevskii \& Kleeorin(2003; 2004b). The solution of (17/1819) will be given for two specific cases. In the first case we apply no restriction to the angular velocity ( the Coriolis number, $\Omega^{*}=2 \Omega \tau_{c}$, is arbitrary) and LSMF is assumed to be weak. In the second case we keep the linear terms in angular velocity and solve eqs.(17/1819) for the case of arbitrary $\beta=\bar{B} /\left(u_{c} \sqrt{\mu \rho}\right)$, where $\bar{B}$ is the strength of the LSMF. In all derivations we keep contributions which are the first order in the shear. Furthermore, for the contributions involving the shear we make two additional simplifications. The first one is that we neglect the density stratification, but leave the contributions of the turbulence intensity stratification. Additionally, we discard the joint effect of the Coriolis force and the shear to the MEMF. In the present study I consider an intermediate nonlinearity which implies that effect of the mean magnetic field and global rotation is not enough strong in order to affect the correlation time of turbulent velocity field.

For integration in k-space I adopt the quasi-isotropic form of the spectra (Roberts and Soward, 1975; Rüdiger and Kichatinov, 1993) for the background turbulence. Additionally, the background magnetic fluctuations are helical, while there is no prescribed kinetic helicity in the background turbulence:

$$
\begin{aligned}
& \hat{v}_{i j}^{(0)}=\left\{\pi_{i j}(\mathbf{k})+\frac{\mathrm{i}}{2 k^{2}}\left(k_{i} \nabla_{j}-k_{j} \nabla_{i}\right)\right\} \frac{\rho^{2} E(k, \mathbf{R})}{8 \pi k^{2}} \\
& \hat{h}_{i j}^{(0)}=\left\{\left(\pi_{i j}(\mathbf{k})+\frac{\mathrm{i}}{2 k^{2}}\left(k_{i} \nabla_{j}-k_{j} \nabla_{i}\right)\right) \frac{\mathcal{B}(k, \mathbf{R})}{8 \pi k^{2}}-\mathrm{i} \varepsilon_{i j p} k_{p} \frac{\mathcal{N}(k, \mathbf{R})}{8 \pi k^{4}}\right\}
\end{aligned}
$$

where, the spectral functions $E(k, \mathbf{R}), \mathcal{B}(k, \mathbf{R}), \mathcal{N}(k, R)$ define, respectively, the intensity of the velocity fluctuations, the intensity of the magnetic fluctuations and amount of current helicity in the background turbulence. They are defined via

$$
\left\langle u^{(0) 2}\right\rangle=\int \frac{E(k, \mathbf{R})}{4 \pi k^{2}} \mathrm{~d}^{3} \mathbf{k},\left\langle b^{(0) 2}\right\rangle=\int \frac{\mathcal{B}(k, \mathbf{R})}{4 \pi k^{2}} \mathrm{~d}^{3} \mathbf{k}, h_{C}^{(0)}=\frac{1}{\mu \rho} \int \frac{\mathcal{N}(k, \mathbf{R})}{4 \pi k^{2}} \mathrm{~d}^{3} \mathbf{k}
$$

where $h_{\mathcal{C}}^{(0)}=\left\langle\mathbf{b}^{(\mathbf{0})} \cdot \nabla \times \mathbf{b}^{(\mathbf{0})}\right\rangle /(\mu \rho)$. In final results we use the relation between intensities of magnetic and kinetic fluctuations which is defined via $\mathcal{B}(k, \mathbf{R})=\varepsilon \mu \bar{\rho} E(k, \mathbf{R})$. The state with $\varepsilon=1$ means equipartition between energies of magnetic an kinetic fluctuations in the background turbulence. The point to note is that inconsistency between (20) and (21) does not influence the final results. The general structure of the mean electromotive force vector obtained within the given framework are in agreement with the known results from the literature (Rädler et al., 2003; Rogachevskii and Kleeorin, 2003). We keep the current helicity contribution in the background turbulence to investigate the nonlinear saturation phase of the helical large-scale dynamo.

The final remarks in this section concern with discussion given in the paper by Rädler and Rheinhardt (2006). There, authors argue that $\tau$ approximation may lead to results which are in conflict with those of SOCA. One difference is apparent between the two approaches: there is no overlap in applicability limits of SOCA and $\tau$ approximation. The given scheme to obtain (17/18/19) is hardly justified for small hydrodynamic Reynolds numbers. The same is true in a highly conductivity limit, where SOCA can be valid only for the small Strouhal numbers. Currently, the range of $\tau$ approximation validity is purely understood. This problem requires further careful study.

There is another reason for difference between results presented in the paper and those of SOCA. In the given variant of $\tau$ approximation the relaxation time $\tau_{c}$ is independent of $\mathbf{k}$. This issue is especially important in computing effects of the nonuniform LSMF and shear. Perhaps, the spectral $\tau$-approximation can correct this defect. For more detail, see (Rädler et al., 2003; Rogachevskii and Kleeorin, 2007). Hense, in confronting MTA and SOCA, it is of some use to simplify the expressions obtained within SOCA by applying the mixing-length approximation. The transition from SOCA to MLT can be done by replacing the spectrum of turbulent fields by the single-scaled function of the form $\delta\left(k-\ell_{c}^{-1}\right) \delta(\omega)$, and applying $\eta k^{2}=\nu k^{2}=\tau_{c}^{-1}$, here $\ell_{c}$ is 
the correlation length of the turbulence. For more details, see (Kichatinov, 1991; Kichatinov et al., 1994).

\section{Results}

\subsection{Weak LSMF, arbitrary Coriolis number}

\subsubsection{Spatially uniform LSMF}

We divide the electromotive force into different contributions, in particular, $\mathcal{E}^{(a)}$ contains the effects of stratification, and $\mathcal{E}^{(s)}$ is due to shear. The contributions due to shear are computed only in slow rotation limit. We find the following expression for $\mathcal{E}^{(a)}$ :

$$
\begin{aligned}
\mathcal{E}^{(a)} & =\left\{(\varepsilon-1)\left(f_{2}^{(a)}(\mathbf{U} \times \overline{\mathbf{B}})+f_{1}^{(a)}(\mathbf{e} \cdot \overline{\mathbf{B}})(\mathbf{e} \times \mathbf{U})\right)+f_{3}^{(a)}(\mathbf{G} \times \overline{\mathbf{B}})\right. \\
& +f_{1}^{(a)}((\mathbf{e} \cdot \mathbf{G})(\mathbf{e} \times \overline{\mathbf{B}})+(\varepsilon-2)(\mathbf{e} \cdot \overline{\mathbf{B}})(\mathbf{e} \times \mathbf{G})) \\
& +f_{4}^{(a)} \mathbf{e}(\mathbf{e} \cdot \overline{\mathbf{B}})(\mathbf{e} \cdot \mathbf{U})+f_{11}^{(a)} \overline{\mathbf{B}}(\mathbf{e} \cdot \mathbf{U})+f_{5}^{(a)} \mathbf{e}(\mathbf{e} \cdot \overline{\mathbf{B}})(\mathbf{e} \cdot \mathbf{G}) \\
& +f_{8}^{(a)}(\mathbf{e}(\overline{\mathbf{B}} \cdot \mathbf{U})+\mathbf{U}(\mathbf{e} \cdot \overline{\mathbf{B}}))+f_{6}^{(a)}(\mathbf{e}(\overline{\mathbf{B}} \cdot \mathbf{G})+\mathbf{G}(\mathbf{e} \cdot \overline{\mathbf{B}}))+f_{10}^{(a)} \overline{\mathbf{B}}(\mathbf{e} \cdot \mathbf{G}) \\
& \left.+f_{9}^{(a)}(\mathbf{e}(\overline{\mathbf{B}} \cdot \mathbf{U})-\mathbf{U}(\mathbf{e} \cdot \overline{\mathbf{B}}))+f_{7}^{(a)}(\mathbf{e}(\overline{\mathbf{B}} \cdot \mathbf{G})-\mathbf{G}(\mathbf{e} \cdot \overline{\mathbf{B}}))\right\}\left\langle u^{(0) 2}\right\rangle \tau_{c} \\
& +2\left\{f_{2}^{(a)} \overline{\mathbf{B}}-f_{1}^{(a)} \mathbf{e}(\mathbf{e} \cdot \overline{\mathbf{B}})\right\} \tau_{c} h_{\mathcal{C}}^{(0)}
\end{aligned}
$$

where functions $f_{\{n\}}^{(a)}=f_{\{n\}}^{(a)}\left(\Omega^{*}, \varepsilon\right)$ (and all which are used below) are given in Appendix A, $\mathbf{U}=\nabla \log \left\langle u^{(0) 2}\right\rangle$ is a scale of the turbulence intensity stratification, $\mathbf{e}=\boldsymbol{\Omega} /|\Omega|$ is a unit vector in direction of global rotation. For the slow rotation limit $\left(\Omega^{*} \rightarrow 0\right)$ we get :

$$
\begin{aligned}
\left.\mathcal{E}^{(a)}\right|_{\Omega^{*} \rightarrow 0} & =\alpha \circ \overline{\mathbf{B}}+\left\langle u^{(0) 2}\right\rangle \tau_{c}\left\{\frac{(\varepsilon-1)}{6}(\mathbf{U} \times \overline{\mathbf{B}})+\frac{\varepsilon}{6}(\mathbf{G} \times \overline{\mathbf{B}})\right\} \\
& +\left\langle u^{(0) 2}\right\rangle \tau_{c} \frac{\Omega^{*}}{12}\{(\varepsilon+2)(((\mathbf{G} \times \mathbf{e}) \times \overline{\mathbf{B}}))+(\varepsilon+1)(((\mathbf{U} \times \mathbf{e}) \times \overline{\mathbf{B}}))\}, \\
\alpha_{i j} & =\delta_{i j} \tau_{c}\left(\left\langle u^{(0) 2}\right\rangle\left\{\frac{2 \varepsilon((\mathbf{e} \cdot \mathbf{U})+(\mathbf{e} \cdot \mathbf{G})) \Omega^{*}}{15}-\frac{2(\mathbf{e} \cdot \mathbf{U}) \Omega^{*}}{5}-\frac{4(\mathbf{e} \cdot \mathbf{G}) \Omega^{*}}{5}\right\}+\frac{h_{\mathcal{C}}^{(0)}}{3}\right) \\
& +\tau_{c}\left\langle u^{(0) 2}\right\rangle \frac{\Omega^{*}}{20}\left\{\left(e_{i} G_{j}+e_{j} G_{i}\right)(\varepsilon+4)+\left(e_{i} U_{j}+e_{j} U_{i}\right)\left(\varepsilon+\frac{11}{3}\right)\right\},
\end{aligned}
$$

where only linear terms in $\Omega$ are kept. Except contributions due to $\mathbf{G}$ equations (24) and (25) are in agreement with results by Rädler et al. (2003) and Brandenburg and Subramanian (2005). The mean transport of the LSMF due to stratification of turbulence is given by second term in (24). They are in agreement with the mixing-length expressions obtained by Kichatinov (1991). Note that, additional components of the turbulent transport may be excited due to the antisymmetric part of $\alpha$-tensor in (25).

For the fast rotation limit $\left(\Omega^{*} \rightarrow \infty\right)$ of (23) we get

$$
\left.\mathcal{E}^{(a)}\right|_{\Omega^{*} \rightarrow \infty} \rightarrow \frac{\pi \tau_{c}}{2}\left(\frac{h_{\mathcal{C}}^{(0)}}{2 \Omega^{*}}-\left\langle u^{(0) 2}\right\rangle\left(\frac{(\mathbf{e} \cdot \mathbf{U})}{2}+(\mathbf{e} \cdot \mathbf{G})\right)\right)(\overline{\mathbf{B}}-\mathbf{e}(\mathbf{e} \cdot \overline{\mathbf{B}})),
$$

where, we keep the next order contribution in $\Omega^{*}$ for the current helicity, as well. The reason for this will be clarified later in section 4. Except the helicity term, eq.(26) is in identical agreement with the mixing-length approximation results obtained by Rüdiger and Kichatinov (1993) within SOCA.

In the case of the spatially uniform LSMF the shear contributions to the mean electromotive force are expressed as follows: 


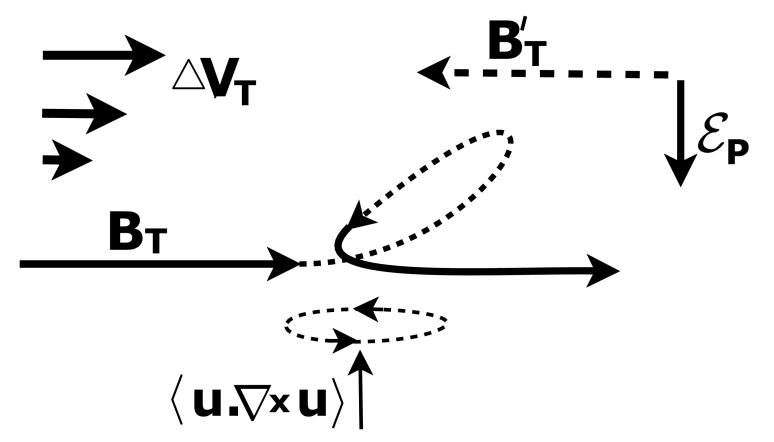

Figure 1: The modification of standard alpha effect (cf. Krause and Rädler (1980)) due to shear. The helical motions (denoted with $\langle\mathbf{u} \cdot \nabla \times \mathbf{u}\rangle$ ) go up, drag and twist the LSMF $\mathbf{B}_{T}$, where index $T$ denotes the toroidal component of LSMF. The shear, $\Delta V_{T}$, additionally, folds the loop in direction of large-scale flow. The effect is equivalent to inducing the transversal large-scale electromotive force, $\mathcal{E}_{P}$ (here index $P$ denotes the poloidal component of the MEMF), and the magnetic field, $\mathbf{B}_{T}^{\prime}$ parallel to original one. Direction of the induced field depends on the sign of the helicity. For the situation given on the picture, the induced field $\mathbf{B}_{T}^{\prime}$ quenches the original LSMF in direction of the gradient of the mean flow. This means that the LSMF is effectively pumped in opposite direction.

$$
\begin{aligned}
\mathcal{E}_{i}^{(s)} & =\varepsilon_{i n m}\left\{A_{4} U_{k} \bar{B}_{n} \bar{V}_{m, k}+A_{2} \bar{B}_{k} \bar{V}_{n, k} U_{m}+A_{3}(\overline{\mathbf{B}} \cdot \mathbf{U}) \bar{V}_{m, n}+A_{1} \bar{V}_{k, n} \bar{B}_{k} U_{m}\right\}\left\langle u^{(0) 2}\right\rangle \\
& +\tau_{c}^{2} \frac{h_{\mathcal{C}}^{(0)}}{2}(\mathbf{W} \times \overline{\mathbf{B}})_{i}-\frac{13}{30} \tau_{c}^{2} h_{\mathcal{C}}^{(0)}\left\{\bar{V}_{n, i}+\bar{V}_{i, n}\right\} \bar{B}_{n},
\end{aligned}
$$

where $\mathbf{W}=\nabla \times \overline{\mathbf{V}}$, we assume that $(\mathbf{U} \cdot \nabla) \overline{\mathbf{V}}=0$ and $A_{1}=(2 \varepsilon-1) \tau_{c}^{2} / 15, A_{2}=-(3 \varepsilon+1) \tau_{r}^{2} / 15$, $A_{3}=(\varepsilon+1) \tau_{c}^{2} / 6, A_{4}=-A_{3}$. Coefficients $A_{1-3}$ correspond to those from Rüdiger and Kichatinov (2006) (hereafter RK06) and $A_{4}$ is corresponding to their $A_{5}$. Recently, similar contributions of the large-scale shear were calculated within SOCA by Rädler and Stepanov (2006) (RS06), as well. We have to note that both the RK06 and RS06 results are related with the case $\varepsilon=0$. The (27) differs with results obtained in RK06 and RS06 papers. For example, after applying the mixing-length relations $\eta k^{2}=\nu k^{2}=\tau_{c}^{-1}$ to expressions given by RK06 we get $A_{1}=\tau_{c}^{2} / 3$ (in our case $-\tau_{c}^{2} / 15$ ) and $A_{2}=-\tau_{c}^{2} / 60$ (compare to our $-\tau_{c}^{2} / 15$ ). Unfortunately RK06 did not present the results for other coefficients. The comparison with RS06 is given in Appendix B. The difference between the given results and those by RK06 and RS06 can be explained, in part, by the crudeness of the given version of tau approximation. Here, we assume that $\tau_{c}$ is independent of $\mathbf{k}$. This especially influences the accuracy of calculations of the contributions due to shear because they involve the derivatives in $\mathbf{k}$ space.

According to (27) the joint effect of current helicity and shear contributes to pumping of LSMF. The interpretation of the effect is difficult to illustrate. To show the general idea we invoke an auxiliary illustration of effect for the helical turbulent motions. It is shown on Fig [1]

\subsubsection{Anisotropic diffusion, the $\Omega \times \mathbf{J}$ and shear-current effects}

In rotating turbulence the magnetic diffusivity become anisotropic (Kichatinov et al., 1994). The corresponding part of the MEMF reads,

$$
\begin{aligned}
\mathcal{E}_{i}^{(d)} & =\left\{\mathrm{f}_{1}^{(d)} e_{n} \bar{B}_{\mathrm{n}, \mathrm{i}}+\mathrm{f}_{2}^{(d)} \varepsilon_{\mathrm{inm}} \bar{B}_{\mathrm{m}, \mathrm{n}}+\varepsilon \mathrm{f}_{3}^{(d)} e_{i} e_{n} e_{m} \bar{B}_{\mathrm{m}, \mathrm{n}}\right. \\
& \left.+\mathrm{f}_{1}^{(a)} \varepsilon_{i n m} e_{n} e_{l}\left(2 \varepsilon \bar{B}_{l, m}-(\varepsilon+1) \bar{B}_{m, l}\right)+\varepsilon \mathrm{f}_{4}^{(d)} e_{n} \bar{B}_{\mathrm{i}, \mathrm{n}}\right\}\left\langle u^{(0) 2}\right\rangle \tau_{c}
\end{aligned}
$$




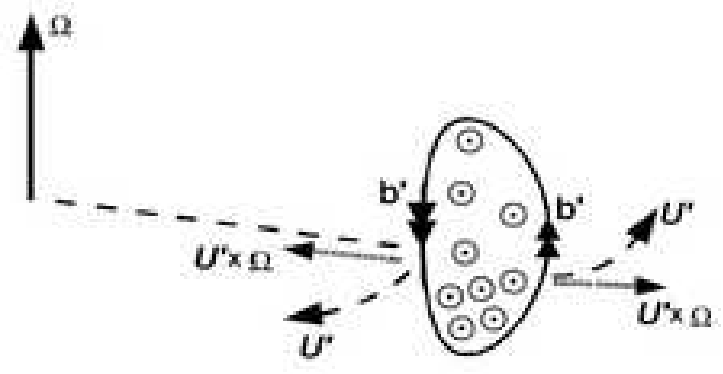

Figure 2: An illustration of $\boldsymbol{\Omega} \times \mathbf{J}$ effect in disk geometry. Direction of rotation is marked by $\boldsymbol{\Omega}$, the large-scale toroidal field has opposite direction to rotational velocity and it is marked by $\odot$, what means that LSMF is perpendicular to the figure's plane and it is directed to the reader. The loop of fluctuating magnetic field, $\mathbf{b}^{\prime}$, comprises LSMF that is nonuniform along the axis of rotation. Its direction is marked by double arrows. The small-scale Lorentz forces induce the azimuthal fluctuations of velocity, $\mathbf{u}^{\prime} \sim\left(\mathbf{b}^{\prime} \cdot \nabla\right) \overline{\mathbf{B}}$. They are marked by dashed lines ending with arrows. The Coriolis force deflects these fluctuations to radial direction (this is marked by dotted lines). The resulting electromotive force has the same direction as the original LSMF and it is proportional to $\left\langle b^{\prime 2}\right\rangle(\Omega \cdot \nabla) \overline{\mathbf{B}}$.

where functions $f_{\{n\}}^{(d)}=f_{\{n\}}^{(d)}\left(\Omega^{*}\right)$ are given in Appendix A. If we put the magnetic fluctuations in background turbulence equal to zero in (28) $(\varepsilon=0)$, we return to results obtained by Kichatinov et al. (1994). The magnetic fluctuation contributions in (28) give rise to the $\boldsymbol{\Omega} \times \mathbf{J}$ effect (see Rädler (1969); Krause and Rädler (1980); Rädler et al. (2003); Kichatinov (2003)) and to additions in anisotropic diffusion. In the slow-rotation limit eq. (28) can be reduced to

$$
\left.\mathcal{E}_{i}^{(d)}\right|_{\Omega * \rightarrow 0}=\left\{e_{n}\left((\varepsilon+5) \bar{B}_{\mathrm{n}, \mathrm{i}}+6 \varepsilon \bar{B}_{\mathrm{i}, \mathrm{n}}\right) \frac{\Omega^{*}}{10}-\varepsilon_{\mathrm{inm}} \bar{B}_{\mathrm{m}, \mathrm{n}}\right\} \frac{\left\langle u^{(0) 2}\right\rangle \tau_{c}}{3},
$$

Eq. (29) corresponds to results by Brandenburg and Subramanian (2005). Note, only magnetic fluctuations contribute to the induction term $(\mathbf{e} \cdot \nabla) \overline{\mathbf{B}}$. The physical interpretation of this effect is straightforward and it is shown on Fig, 2

Lets consider the situation in disk geometry and the rotating media penetrated by the inhomogeneous toroidal LSMF. For simplicity, we assume that LSMF is nonuniform along the axis of rotation. Let the direction of LSMF be opposite to direction of rotating plasma. If the loop of the small-scale fluctuating magnetic field comprises LSMF, it induces fluctuation of velocity in azimuthal direction. The influence of the Coriolis force declines the velocities in radial direction. The effective electromotive force is co-lined with original LSMF and is proportional to $\left\langle b^{2}\right\rangle(\Omega \cdot \nabla) \overline{\mathbf{B}}$, see Fig 2.

The shear-current effect Rogachevskii and Kleeorin (2003) (hereafter RK03) is of similar nature because the large-scale vorticity $\mathbf{W}=\nabla \times \overline{\mathbf{V}}$ and the Coriolis force act on the turbulent motions in a like manner. The additional contributions due to shear in the diffusion part of the mean electromotive force are expressed as follows,

$$
\mathcal{E}_{i}^{(V)}=\varepsilon_{i n m}\left\{C_{2} \bar{B}_{\mathrm{n}, 1} \bar{V}_{m, \mathrm{l}}+C_{1} \bar{V}_{\mathrm{l}, \mathrm{m}} \bar{B}_{\mathrm{n}, \mathrm{l}}+C_{3} \bar{V}_{l, \mathrm{~m}} \bar{B}_{\mathrm{l}, \mathrm{n}}+C_{4} \bar{B}_{\mathrm{l}, \mathrm{n}} \bar{V}_{\mathrm{m}, \mathrm{l}}\right\}\left\langle u^{(0) 2}\right\rangle,
$$

where $C_{1}=(\varepsilon-3 / 5) \tau_{c}^{2} / 6, C_{2}=(\varepsilon-1) \tau_{c}^{2} / 5, C_{3}=(1+\varepsilon) \tau_{c}^{2} / 15, C_{4}=-(7 \varepsilon+11) \tau_{c}^{2} / 30$. Coefficients $C_{1-4}$ correspond to those from RK06. After applying the mixing-length approximation to RK06's results we get $C_{1}=-2 \tau_{c}^{2} / 5, C_{2}=-4 \tau_{c}^{2} / 15, C_{3}=0, C_{4}=-\tau_{c}^{2} / 5$. In confronting these coefficients to ours, we see the difference. It can be explained, in part, by the crudeness of the given variant of tau approximation. The comparison with RS06 is given in Appendix B. As shown by Rogachevskii and Kleeorin (2007) the spectral $\tau$ approximation is capable to give result in closer agreement with those of SOCA. 
In the commonly accepted scheme of the solar $\alpha \Omega$ dynamo, the poloidal LSMF of the Sun is produced from the large-scale toroidal magnetic field via the alpha effect. Expressions (28 30) hold contributions which are capable to induce the MEMF along the LSMF and consequently these terms are potentially very important for the solar dynamo because they provides additional induction sources of the large-scale poloidal magnetic field of the Sun. Below, I consider the efficiency of induction effect along the nonuniform LSMF due to global rotation and shear.

For the sake of simplicity we restrict consideration to the axisymmetric LSMF in the Keplerian disk in the disk geometry. In cylindrical coordinates $(r, \phi, z)$ the axisymmetric LSMF can be expressed via $\overline{\mathbf{B}}=B \mathbf{e}_{\phi}+\operatorname{rot}\left(A \mathbf{e}_{\phi}\right)$ and the global rotation velocity is $\overline{\mathbf{V}}=r \Omega \mathbf{e}_{\phi}$. We assume that toroidal LSMF exceeds its poloidal counterpart, $\overline{\mathbf{B}} \approx B \mathbf{e}_{\phi}$. In (2830) we leave only those terms that induce the toroidal MEMF and skip the usual contributions due to turbulent diffusion as well.

In the Keplerian disk we have $\partial \log \Omega / \partial \log r=-3 / 2$. For the given conditions the contribution of shear in 30 is defined by terms at $C_{3}, C_{4}$. It is calculated as follows

$$
\mathcal{E}_{\phi}^{(V)} \approx\left(C_{3}\left(\nabla_{r} \overline{\mathbf{V}}\right)_{\phi}\left(\nabla_{z} \overline{\mathbf{B}}\right)_{\phi}+C_{4}\left(\nabla_{z} \overline{\mathbf{B}}\right)_{\phi}\left(\nabla_{\phi} \bar{V}\right)_{r}\right)\left\langle u^{(0) 2}\right\rangle
$$

where covariant derivatives are $\left(\nabla_{r} \overline{\mathbf{V}}\right)_{\phi}=r \partial_{r}\left(\bar{V}_{\phi} / r\right),\left(\nabla_{\phi} \overline{\mathbf{V}}\right)_{r}=-\bar{V}_{\phi} / r$ and $\left(\nabla_{z} \overline{\mathbf{B}}\right)_{\phi}=\partial_{z} B$. Then, the contribution of shear to the MEMF is defined by $r \partial_{r}\left(\bar{V}_{\phi, r} / r\right) \tau_{c}=-3 \Omega^{*} / 4$ and $-\tau_{c} \bar{V}_{\phi} / r=-.5 \Omega^{*}$. Our derivations are valid in the case of the weak shear flow, $\left|V_{i, j} \tau_{c}\right| \ll 1$. For the Keplerian disks this condition is fulfilled if $\Omega^{*} \ll 1$. In taking the latter into account and using (29), we find the azimuthal component of the MEMF generated from the non-uniform toroidal component of LSMF via effects of the global rotation and shear,

$$
\mathcal{E}_{\phi} \approx \frac{2 \Omega^{*}}{15}(2 \varepsilon+1)\left\langle u^{(0) 2}\right\rangle \tau_{c} \frac{\partial B}{\partial z} .
$$

Therefore if the LSMF is concentrated to the plan of disk the induced MEMF is in direction of the LSMF.

\subsection{Slow rotation, arbitrary LSMF}

\subsubsection{Spatially uniform LSMF}

In this part of the paper we consider results obtained for the slow rotation limit. In what follows, no restriction is applied to the strength of the LSMF. The MEMF, that is induced due to influence of rotation and stratification on the turbulence, is described with expression

$$
\begin{aligned}
\mathcal{E}^{(a)} & =\left\langle u^{(0) 2}\right\rangle \tau_{c}\left\{\varphi_{1}^{(a)}(\mathbf{G} \times \overline{\mathbf{B}})+\varphi_{2}^{(a)}(\mathbf{U} \times \overline{\mathbf{B}})+\tau_{c}(\boldsymbol{\Omega} \cdot \overline{\mathbf{B}})\left(\varphi_{4}^{(a)} \mathbf{G}+\varphi_{10}^{(a)} \mathbf{U}\right)\right. \\
& +\tau_{\mathbf{c}} \overline{\mathbf{B}}\left(\varphi_{\mathbf{6}}^{(\mathbf{a})}(\boldsymbol{\Omega} \cdot \mathbf{G})+\varphi_{\mathbf{8}}^{(\mathbf{a})}(\boldsymbol{\Omega} \cdot \mathbf{U})\right)+\tau_{c} \boldsymbol{\Omega}\left(\varphi_{5}^{(a)}(\overline{\mathbf{B}} \cdot \mathbf{G})+\varphi_{9}^{(a)}(\overline{\mathbf{B}} \cdot \mathbf{U})\right) \\
& \left.+\tau_{\mathbf{c}} \frac{(\boldsymbol{\Omega} \cdot \overline{\mathbf{B}}) \overline{\mathbf{B}}}{\overline{\mathbf{B}}^{\mathbf{2}}}\left(\varphi_{\mathbf{3}}^{(\mathbf{a})}(\overline{\mathbf{B}} \cdot \mathbf{G})+\varphi_{\mathbf{7}}^{(\mathbf{a})}(\overline{\mathbf{B}} \cdot \mathbf{U})\right)\right\}+\tau_{c} h_{\mathcal{C}}^{(0)} \varphi_{1}^{(h)} \overline{\mathbf{B}}
\end{aligned}
$$

where $\varphi_{n}^{(a)}$ are functions of $\beta$ defined in the appendix. This formula generalizes the similar results by Rüdiger and Kichatinov (1993); Kichatinov and Rüdiger (1992) taking the density stratification, magnetic fluctuations and current helicity into account. The nonlinear MEMF of helical MHD turbulence was considered by Rogachevskii and Kleeorin (2004a), as well. For the strong LSMF limit we obtain

$$
\begin{aligned}
\left.\mathcal{E}^{(a)}\right|_{\beta \rightarrow \infty} & =\left\{\frac{\tau_{c}}{8}\left((\varepsilon+1)(\overline{\mathbf{B}} \cdot \mathbf{U})+\frac{3(3 \varepsilon+5)}{8}(\overline{\mathbf{B}} \cdot \mathbf{G})\right)\left(\boldsymbol{\Omega}-\frac{(\boldsymbol{\Omega} \cdot \overline{\mathbf{B}}) \overline{\mathbf{B}}}{\bar{B}^{2}}\right)\right. \\
& \left.+\frac{3 \varepsilon+1}{64}(\mathbf{G} \times \overline{\mathbf{B}})\right\} \frac{\pi}{\beta}\left\langle u^{(0) 2}\right\rangle \tau_{c} .
\end{aligned}
$$




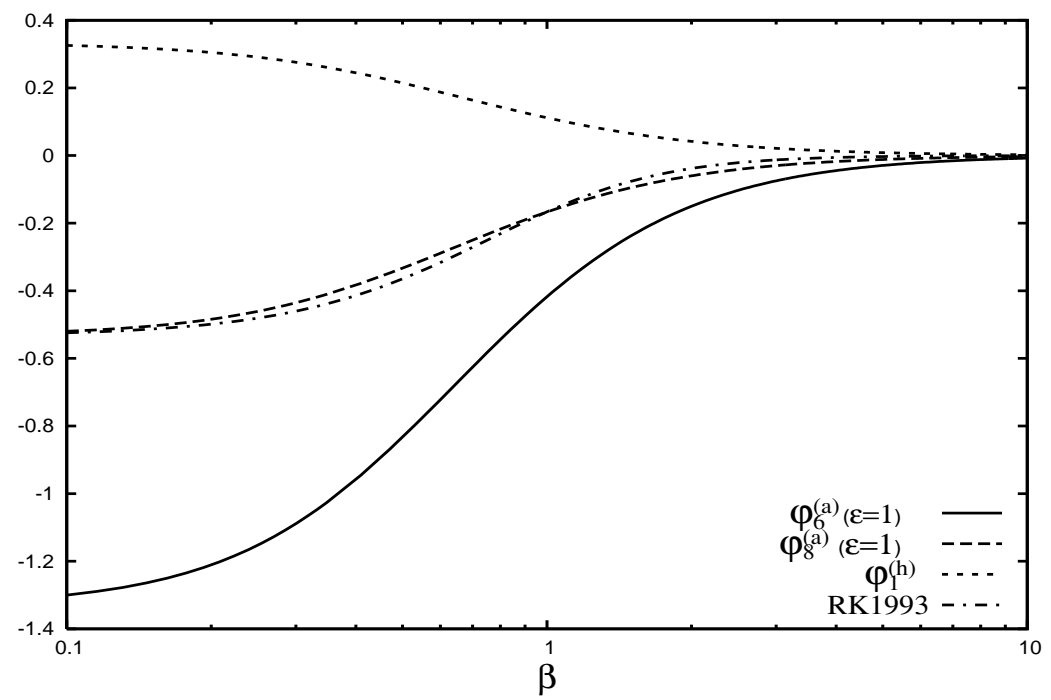

Figure 3: The quenching functions for isotropic components of $\alpha$-effect.

The results by Rüdiger and Kichatinov (1993) can be recovered from (34), if we put $G=0$ and $\varepsilon=0$. Following to arguments given in the paper cited above, we conclude that the MEMF like (34) does not produce a dynamo.

The first term at the upper line of (33) describes the so-called "turbulent buoyancy" Kichatinov and Rüdiger (1992)). The expression (34) shows that the transport of LSMF is downward for the strong magnetic field limit. For the case of the weak field we get $\varphi_{1}^{(a)} \approx \varepsilon / 6+\left(6 \varepsilon-8 \beta^{2}\right) / 15$. Then, if we neglect contributions due to small-scale magnetic fluctuations, we obtain that for the weak field transport is upward (opposite to direction of $\mathbf{G}$ ). In this case the effective drift velocity is proportional to the LSMF's pressure (Kichatinov and Rüdiger (1992)). In this aspect it is similar to the usual buoyancy of magnetic flux tubes (Parker (1979)). Furthermore, we find that the large-scale inhomogeneity of magnetic fluctuations provide the downward drift of LSMF in the whole range of magnetic field strength.

The quenching functions for the isotropic components of $\alpha$ effect are shown on Fig 3 There, for comparison, via the dash-dotted line, we show the curve corresponding to quenching of isotropic components of $\alpha$ effect obtained within SOCA in (Rüdiger and Kichatinov (1993)).

In the strong LSMF limit we found that $\alpha$ effect is quenched as $\beta^{-2}$ which is different from results by Rüdiger and Kichatinov (1993) and similar to findings by Rogachevskii and Kleeorin (2004a). Though, as seen from the figure, the numerical difference between the quenching curves obtained within SOCA (dash-dotted line) and MTA (dashed line) is within a few percents.

The non-linear electromotive force induced by shear is expressed as follows,

$$
\begin{aligned}
\mathcal{E}_{i}^{(s)} & =\varepsilon_{i n m}\left\{\varphi_{1}^{(s)} \frac{\bar{B}_{l} \bar{B}_{k}}{\bar{B}^{2}} \bar{V}_{l, k} U_{n} \bar{B}_{m}+\varphi_{2}^{(s)} \bar{B}_{l} \bar{V}_{l, m} U_{n}+\varphi_{3}^{(s)}(\mathbf{U} \cdot \overline{\mathbf{B}}) \frac{\bar{B}_{l} \bar{B}_{m}}{\bar{B}^{2}}\left(\bar{V}_{l, n}-\bar{V}_{n, l}\right)\right. \\
& \left.+\varphi_{4}^{(s)} U_{l} \bar{V}_{n, l} \bar{B}_{m}+\varphi_{5}^{(s)} U_{n} \bar{V}_{l, m} \bar{B}_{l}\right\}\left\langle u^{(0) 2}\right\rangle \tau_{c}^{2} \\
& +\tau_{c}^{2} h_{\mathcal{C}}^{(0)}\left\{\varphi_{4}^{(h)} \bar{V}_{m, n} \frac{\bar{B}_{m} \bar{B}_{n}}{\bar{B}^{2}} \bar{B}_{i}+\varphi_{3}^{(h)}\left(\bar{V}_{n, i}+\bar{V}_{i, n}\right) \bar{B}_{n}+\varphi_{2}^{(h)}(\mathbf{W} \times \overline{\mathbf{B}})_{i}\right\}
\end{aligned}
$$

From the structure of (35) we can conclude that contributions with $\varphi_{2,3,5}^{(s)}$ and the second term in brackets with $\varphi_{5}^{(5)}$ can be interpreted as the $\alpha$ effect. The terms with $\varphi_{1,4}^{(s)}$ and $\varphi_{2}^{(h)}$ provide the pumping of LSMF. Surprisingly, the $\alpha$-effect like terms survive even in the limit of the strong 


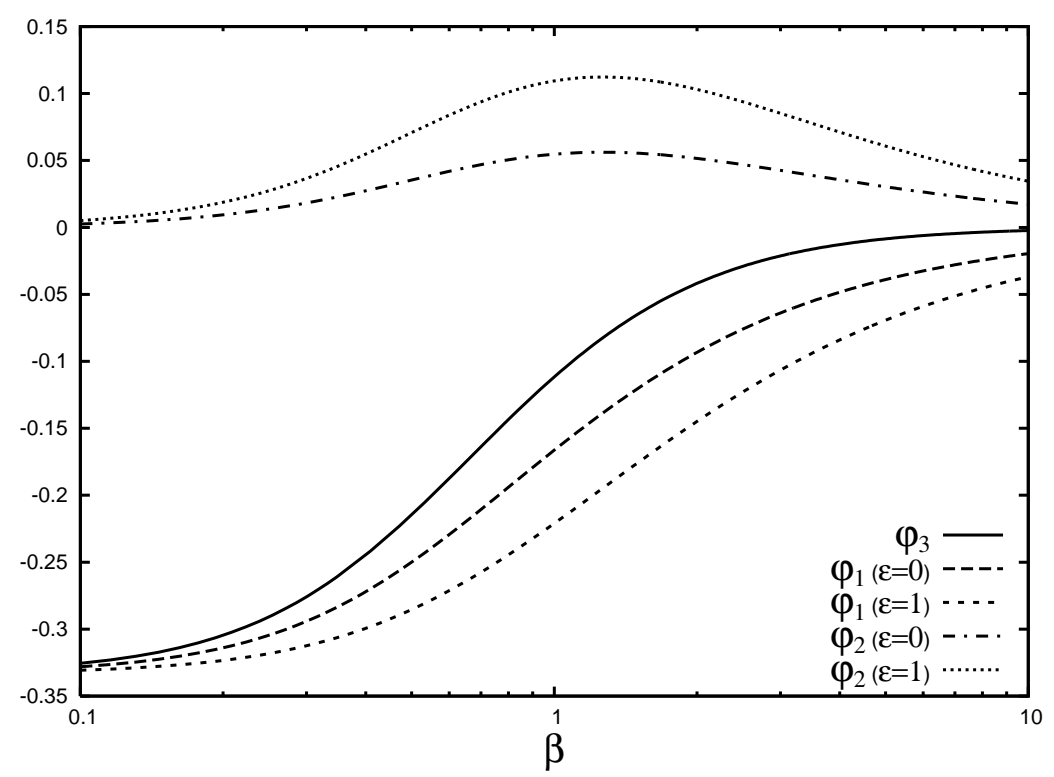

Figure 4: Functions defining the nonlinear turbulent diffusion of LSMF (see eq (37)).

magnetic field. In this case we get

$$
\begin{aligned}
\left.\mathcal{E}_{i}^{(s)}\right|_{\beta \rightarrow \infty} & =\varepsilon_{i n m}\left\{\frac{3}{4}(\varepsilon-1)\left(\frac{\bar{B}_{l} \bar{B}_{k}}{\bar{B}^{2}} \bar{V}_{l, k} U_{n} \bar{B}_{m}-\bar{B}_{l} \bar{V}_{l, m} U_{n}\right)\right. \\
& \left.+(\varepsilon+1)(\mathbf{U} \cdot \overline{\mathbf{B}}) \frac{\bar{B}_{l} \bar{B}_{m}}{\bar{B}^{2}}\left(\bar{V}_{l, n}-\bar{V}_{n, l}\right)\right\} \frac{\pi}{16 \beta}\left\langle u^{(0) 2}\right\rangle \tau_{c}^{2} \\
& +\frac{3 \pi}{64 \beta} \tau_{c}^{2} h_{\mathcal{C}}^{(0)}\left\{\bar{V}_{m, n} \frac{\bar{B}_{m} \bar{B}_{n}}{\bar{B}^{2}} \bar{B}_{i}-\left(\bar{V}_{n, i}+\bar{V}_{i, n}\right) \bar{B}_{n}+(\mathbf{W} \times \overline{\mathbf{B}})_{i}\right\},
\end{aligned}
$$

According to (27) and (36) the pumping of the LSMF due to joint effect of current helicity and shear have the same sign for the weak and strong LSMF.

\subsubsection{Diffusion, $\Omega \times \mathbf{J}$ and shear current effect}

The results for nonlinear turbulent diffusion are similar to those found within SOCA by Kichatinov et al. (1994). We have

$$
\mathcal{E}^{(d)}=\left\{\varphi_{3} \nabla \times \overline{\mathbf{B}}+\left(\varphi_{2} \frac{((\nabla \times \overline{\mathbf{B}}) \times \overline{\mathbf{B}})}{\bar{B}^{2}}+\varphi_{1} \nabla \log \left(\frac{\bar{B}^{2}}{2}\right)\right) \times \overline{\mathbf{B}}\right\}\left\langle u^{(0) 2}\right\rangle \tau_{c}+\mathcal{E}^{(w)},
$$

where $\mathcal{E}^{(w)}$ stands for the contributions due to rotation. The corresponding quenching functions are given on Fig, 4

The next formula generalizes the results for the nonlinear diffusion of LSMF to the case of the slowly rotating media,

$$
\begin{aligned}
\mathcal{E}_{i}^{(w)} & =\left\{\varphi_{8}^{(w)} \nabla_{i}(\boldsymbol{\Omega} \cdot \overline{\mathbf{B}})+\varphi_{1}^{(w)} \frac{(\boldsymbol{\Omega} \cdot \overline{\mathbf{B}})}{2} \nabla_{i} \log \left(\bar{B}^{2}\right)+\varphi_{4}^{(w)} \bar{B}_{i} \frac{(\overline{\mathbf{B}} \cdot \nabla)(\boldsymbol{\Omega} \cdot \overline{\mathbf{B}})}{\bar{B}^{2}}\right. \\
& +\varphi_{5}^{(w)} \Omega_{i} \frac{(\overline{\mathbf{B}} \cdot \nabla)}{2} \log \left(\bar{B}^{2}\right)+\varphi_{3}^{(w)} \bar{B}_{i} \frac{(\Omega \cdot \overline{\mathbf{B}})}{\bar{B}^{2}} \frac{(\overline{\mathbf{B}} \cdot \nabla)}{2} \log \left(\bar{B}^{2}\right) \\
& \left.+\varphi_{6}^{(w)} \frac{(\Omega \cdot \overline{\mathbf{B}})}{\bar{B}^{2}}(\overline{\mathbf{B}} \cdot \nabla) \bar{B}_{i}+\varphi_{2}^{(w)} \bar{B}_{i} \frac{(\Omega \cdot \nabla)}{2} \log \left(\bar{B}^{2}\right)+\varphi_{7}^{(w)}(\boldsymbol{\Omega} \cdot \nabla) \bar{B}_{i}\right\}\left\langle u^{(0) 2}\right\rangle \tau_{c}^{2} .
\end{aligned}
$$




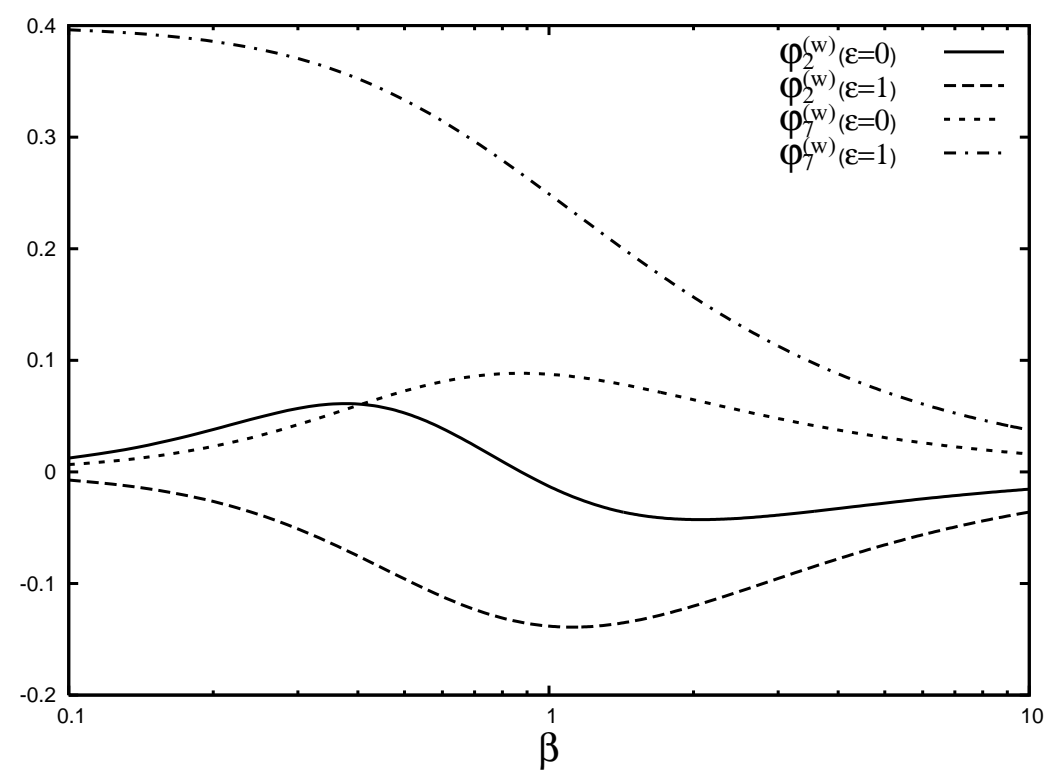

Figure 5: The quenching functions for " $\Omega \times \mathbf{J}$ " generation effect for different parameters .

The last two terms at the third line in (38) are related with the generation of MEMF along the direction of LSMF. The corresponding functions $\varphi_{2}^{(w)}$ and $\varphi_{7}^{(w)}$ are shown on Fig.5. As can be seen there, in the absence of the background magnetic fluctuations $(\varepsilon=0)$ the generation due to $\Omega \times \mathbf{J}$-effect exists only in nonlinear regime.

If $\beta>1$, functions $\varphi_{2}^{(w)}$ and $\varphi_{7}^{(w)}$ have opposite signs everywhere. Note, while the term $(\boldsymbol{\Omega} \cdot \nabla) \bar{B}_{i}$ induces MEMF in direction of LSMF's gradients along axis of rotation, the term $\bar{B}_{i}(\Omega \cdot \nabla) \log \left(\bar{B}^{2}\right)$ induces MEMF in opposite direction. Formally, the latter effect is similar to $\alpha$-effect. The only difference with the standard $\alpha$ is that instead stratification parameters of turbulence we have a parameter which is related with nonuniform distribution of the LSMF's energy. For the solar magnetic fields the effect is antisymmetric about equator. Below, it is shown that in the strong LSMF this $\alpha$ is quenched by factor $\beta^{-1}$ which is lesser than for standard $\alpha$.

For the limit of the strong LSMF we get

$$
\begin{aligned}
\left.\mathcal{E}_{i}^{(w)}\right|_{\beta \rightarrow \infty} & =\left\{(17 \varepsilon+47)\left(\nabla_{i}(\boldsymbol{\Omega} \cdot \overline{\mathbf{B}})-\frac{(\boldsymbol{\Omega} \cdot \overline{\mathbf{B}})}{2} \nabla_{i} \log \left(\bar{B}^{2}\right)\right)\right. \\
& -(21 \varepsilon+43)\left(\Omega_{i} \frac{(\overline{\mathbf{B}} \cdot \nabla)}{2} \log \left(\bar{B}^{2}\right)+\bar{B}_{i} \frac{(\overline{\mathbf{B}} \cdot \nabla)(\Omega \cdot \overline{\mathbf{B}})}{\bar{B}^{2}}+\frac{(\Omega \cdot \overline{\mathbf{B}})}{\bar{B}^{2}}(\overline{\mathbf{B}} \cdot \nabla) \bar{B}_{i}\right) \\
& +3(21 \varepsilon+43) \frac{(\Omega \cdot \overline{\mathbf{B}})(\overline{\mathbf{B}} \cdot \nabla)}{2 \bar{B}^{2}} \log \left(\bar{B}^{2}\right) \bar{B}_{i} \\
& \left.-(37 \varepsilon+27)\left(\bar{B}_{i} \frac{(\Omega \cdot \nabla)}{2} \log \left(\bar{B}^{2}\right)-(\boldsymbol{\Omega} \cdot \nabla) \bar{B}_{i}\right)\right\} \frac{\pi}{512 \beta}\left\langle u^{(0) 2}\right\rangle \tau_{c}^{2} .
\end{aligned}
$$

From there we find that $\Omega \times \mathbf{J}$-effect maintain the generation part of the MEMF even for the strong LSMF. The amplitude of effect tends to constant as the strength of LSMF is increased. It hardly possible to make a definite conclusion about the dynamo effect in this case because the generation part of (39) is contributed by terms with opposite signs.

The MEMF's contributions due to shear are defined by, 


$$
\begin{aligned}
\mathcal{E}_{i}^{(V)} & =\left\langle u^{(0) 2}\right\rangle \tau_{c}^{2} \varepsilon_{i n m}\left\{\varphi_{1}^{(V)} \bar{V}_{n, l} \bar{B}_{l, m}+\frac{\bar{B}_{l} \bar{B}_{m}}{\bar{B}^{2}} \bar{V}_{l, k}\left(\varphi_{2}^{(V)} \bar{B}_{k, n}+\varphi_{3}^{(V)} \bar{B}_{k, m}\right)\right. \\
& +\left(\varphi_{4}^{(V)} \bar{V}_{n, l}+\varphi_{5}^{(V)} \bar{V}_{l, n}\right) \bar{B}_{m, l}+\varphi_{6}^{(V)} \frac{\bar{B}_{l} \bar{B}_{n}}{\bar{B}^{2}} \bar{V}_{l, k} \bar{B}_{m, k}+\varphi_{7}^{(V)} \frac{\bar{B}_{k} \bar{B}_{l}}{\bar{B}^{2}} \bar{V}_{l, k} \bar{B}_{m, n} \\
& \left.+\varphi_{8}^{(V)} \frac{\bar{B}_{k} \bar{B}_{l}}{\bar{B}^{2}} \bar{V}_{m, n} \bar{B}_{l, k}+\varphi_{9}^{(V)} \bar{V}_{l, n} \bar{B}_{l, m}+\frac{\bar{B}_{k} \bar{B}_{l}}{\bar{B}^{2}} \bar{V}_{l, n}\left(\varphi_{10}^{(V)} \bar{B}_{k, m}+\varphi_{11}^{(V)} \bar{B}_{m, k}\right)\right\},
\end{aligned}
$$

where, for the sake of simplicity, we leave only the largest contributions and those which are important for the solar-type dynamo models, where the strength of LSMF component along direction of the large-scale flow dominates components directed along the shear. Reader can find the expressions for $\varphi_{n}^{(V)}$ in Appendix A. The full expression has a much more complicated tensorial structure than (40). In the case of the strong LSMF we get,

$$
\begin{aligned}
\left.\mathcal{E}_{i}^{(V)}\right|_{\beta \rightarrow \infty} & =\frac{\tau_{c}^{2}}{6}\left\langle u^{(0) 2}\right\rangle \varepsilon_{i n m}\left\{\left(\frac{\varepsilon+15}{20} \bar{V}_{n, l}-\frac{\varepsilon}{5} \bar{V}_{l, n}\right) \bar{B}_{m, l}+(\varepsilon+1) \frac{\bar{B}_{l} \bar{B}_{n}}{\bar{B}^{2}} \bar{V}_{l, k} \bar{B}_{m, k}\right. \\
& -\frac{3 \varepsilon+13}{20} \bar{V}_{n, l} \bar{B}_{l, m}-\frac{\varepsilon+3}{2} \frac{\bar{B}_{l} \bar{B}_{m}}{\bar{B}^{2}} \bar{V}_{l, k} \bar{B}_{k, m}+\frac{\varepsilon+1}{10} \bar{V}_{l, n} \bar{B}_{l, m} \\
& \left.+\frac{\bar{B}_{k} \bar{B}_{l}}{\bar{B}^{2}} \bar{V}_{l, n}\left((\varepsilon+1) \bar{B}_{m, k}-\frac{\varepsilon+3}{4} \bar{B}_{k, m}\right)\right\}
\end{aligned}
$$

Now, we would like to consider efficiency of induction effect along the nonuniform LSMF due to global rotation and shear in nonlinear regimes for the Keplerian discs. As before, we assume a disc penetrated by the large-scale toroidal magnetic field that is nonuniform along the axis of rotation. From (39|40) we get

$$
\mathcal{E}_{\phi} \approx \frac{\Omega^{*}}{2}\left\langle u^{(0) 2}\right\rangle \tau_{c} \varphi^{(w V)} \frac{\partial B}{\partial z}
$$

where the quenching function is $\varphi^{(w V)}=\varphi_{2}^{(w)}+\varphi_{7}^{(w)}+1.5\left(\varphi_{9}^{(V)}+\varphi_{10}^{(V)}\right)+\varphi_{1}^{(V)}$. Note, eq. (42) transforms to eq.(32) in limit $\beta \rightarrow 0$. The dependence of $\varphi^{(w V)}$ on the LSMF's strength is shown on the Fig, 6 ,

Results given on the Fig 6 show that the $\varphi^{(w V)}$ is positive for $\beta<1$ and negative for $\beta>1$ for all $\varepsilon$. This result supports an idea about the change of dynamo type in passing from linear to non-linear regime of the LSMF's generation by $\Omega \times \mathbf{J}$ and shear-current effects. Previously we found that the induction term due to $\Omega \times \mathbf{J}$ effect tends to constant when $\beta \rightarrow \infty$ (eq.(39)) while the induction term due to shear-current effect is growing under $\beta \rightarrow \infty$ (eq. (411)). Therefore, the primary nonlinear generation effect in the differentially rotating uniform MHD turbulence penetrated by the nonuniform toroidal LSMF may be due to shear-current effect. The same nonlinear dependence of shear-current effect was discovered in the paper by Rogachevskii and Kleeorin (2004a) for the different kind of MTA. In the next section I show that the given sources of the MEMF ultimately result to current helicity generation. Therefore, the effect considered above is saturated dynamically due to magnetic helicity conservation law.

\section{The current helicity evolution}

As we have seen, the current helicity contributes to the different kind of MEMF's action, not only to the $\alpha$ effect. The recent papers (Subramanian and Brandenburg, 2004) show that the magnetic helicity conservation law can be described in terms of the current helicity evolution if the assumption of the scale separation is fulfilled. For the time being the redistribution of current helicity over the space scales is not satisfactory understood. One attempt to describe the helicity evolution in turbulent media penetrated by LSMF was given in the papers by Brandenburg and Subramanian 


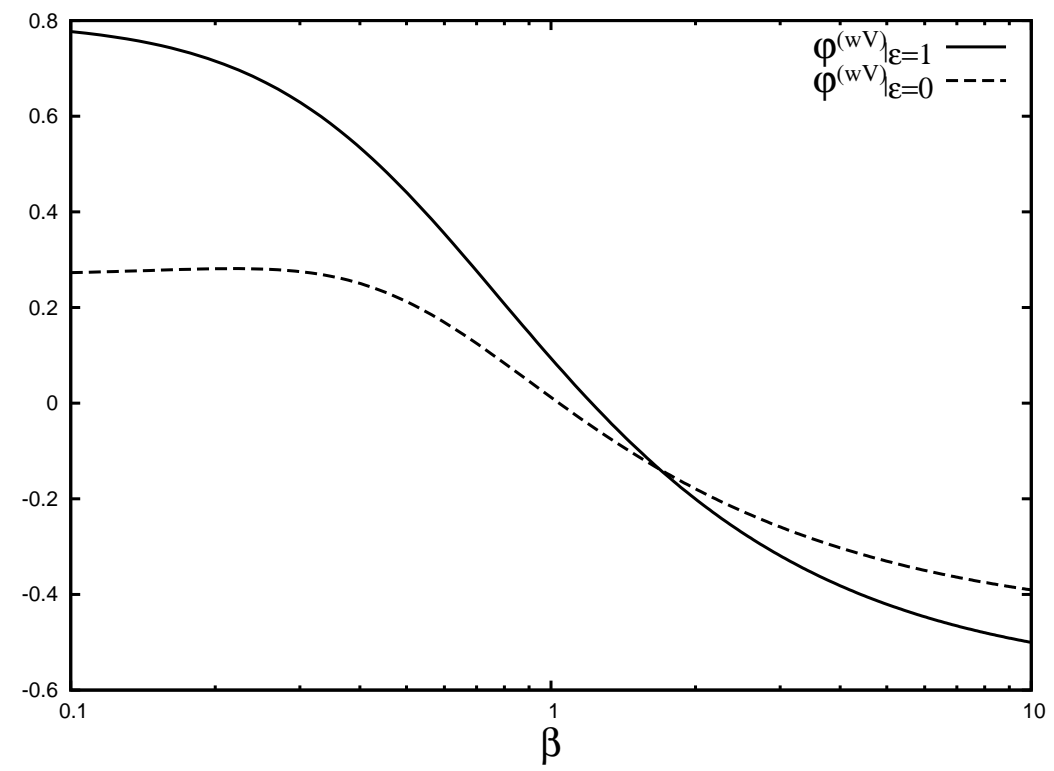

Figure 6: The dependence of induction effect along the nonuniform toroidal LSMF on the strength of magnetic field.

(2005); Subramanian and Brandenburg (2004). Here, we will follow their results and obtain the explicit evolutionary equation for the current helicity. The equation in question can be derived from (34). After integration over the large-scale variables we can get the general equation for the current helicity in the following form,

$$
\begin{aligned}
\frac{\partial h_{\mathcal{C}}}{\partial t} & =-\frac{h_{\mathcal{C}}}{\tau_{h}}+\frac{2}{\mu \rho} \varepsilon_{p l m} \int\left[k^{2} \hat{\varkappa}_{l p} \frac{\bar{B}_{m}}{\rho}-\mathrm{i} \hat{\varkappa}_{l p}(\mathbf{k} \cdot \nabla)\left(\frac{\bar{B}_{m}}{\rho}\right)-\frac{\mathrm{i}}{2}(\mathbf{k} \cdot \nabla)\left(\hat{\varkappa}_{l p} \frac{\bar{B}_{m}}{\rho}\right)\right. \\
& \left.+\mathrm{i} k_{p} \nabla_{n}\left(\hat{\varkappa}_{l n} \frac{\bar{B}_{m}}{\rho}\right)+\frac{1}{2} \bar{V}_{l, n}\left(\mathrm{i} k_{p}-\frac{1}{2} \nabla_{p}\right)\left(\hat{h}_{m n}-\hat{h}_{n m}\right)-\frac{1}{2} \bar{V}_{l, m} \nabla_{n} \hat{h}_{n p}\right] \mathrm{d} \mathbf{k} .
\end{aligned}
$$

The third order moments were replaced by $-h_{\mathcal{C}} / \tau_{h}, \tau_{h}$ - is a relaxation time for the current helicity . This is a rather rough way because the triple correlations may give important contribution for the helicity redistribution over the space scales (Frisch et al., 1975; Kleeorin and Ruzmaikin, 1982; Kleeorin and Rogachevskii, 1999). Because of the very rough assumptions used in derivation of (43), it should be considered with caution. In spite of the latter, the equation (43) provides a useful tool for investigation the nonlinear saturation in helical mean-field dynamo Brandenburg and Subramanian, 2004). Except for contributions due to density stratification and shear, equation (43) can be reproduced from results of BS05 after substitution identity $\varepsilon_{i j k} \varepsilon_{i p q} \varepsilon_{q l m}=\varepsilon_{l m k} \delta_{j p}-\varepsilon_{l m j} \delta_{k p}$ in eq. (10.71) there. Inspection of (43) shows that if we replace $k^{2} \rightarrow \ell_{c}^{-2}$ and use (12), we can write the evolutionary equation in the following form,

$$
\begin{aligned}
\frac{\partial h_{\mathcal{C}}}{\partial t} & =-\frac{2(\mathcal{E} \cdot \overline{\mathbf{B}})}{\mu \rho \ell_{c}^{2}}-\frac{h_{\mathcal{C}}}{\tau_{h}}+\frac{2}{\mu \rho} \varepsilon_{p l m} \int\left[-\mathrm{i} \hat{\varkappa}_{l p} k^{n} \nabla_{n}\left(\frac{\bar{B}_{m}}{\rho}\right)-\frac{\mathrm{i}}{2}(\mathbf{k} \cdot \nabla)\left(\hat{\varkappa}_{l p} \frac{\bar{B}_{m}}{\rho}\right)\right. \\
& \left.+\mathrm{i} k_{p} \nabla_{n}\left(\hat{\varkappa}_{l n} \frac{\bar{B}_{m}}{\rho}\right)+\frac{1}{2} \bar{V}_{l, n}\left(\mathrm{i} k_{p}-\frac{1}{2} \nabla_{p}\right)\left(\hat{h}_{m n}-\hat{h}_{n m}\right)-\frac{1}{2} \bar{V}_{l, m} \nabla_{n} \hat{h}_{n p}\right] \mathrm{d} \mathbf{k} .
\end{aligned}
$$

According to (Frisch et al., 1975; Kleeorin and Ruzmaikin, 1982; Vainshtein, 1983; Brandenburg, 2001; Vishniac and Cho, 2001) the first term in (44) is responsible for helicity generation in turbulent medium. The rest part of equation can be interpreted as the helicity fluxes (Vishniac and Cho, 2001; Subramanian and Brandenburg, 2004, 2005). The given expression for helicity fluxes is incomplete because the contribution of the third order moments is dropped in (44). As the first step 
we consider the case of the weak LSMF. From (44) and (18/17/19) we get

$$
\begin{aligned}
\frac{\partial h_{\mathcal{C}}}{\partial t}+\frac{1}{\tau_{h}} h_{\mathcal{C}} & =-\frac{2}{\mu \rho \ell_{c}^{2}}(\mathcal{E} \cdot \overline{\mathbf{B}})+\frac{(\varepsilon-1)}{\mu \rho \tau_{c}}\left\{2 f_{1}^{(a)}(\mathbf{e} \cdot \overline{\mathbf{B}})(\mathbf{e} \cdot(\mathbf{U} \times \overline{\mathbf{B}}))\right. \\
& +\frac{(\mathbf{e} \cdot \mathbf{G})}{3}\left(f_{4}^{(d)} \bar{B}^{2}+f_{3}^{(d)}(\mathbf{e} \cdot \overline{\mathbf{B}})^{2}\right)+2 f_{2}^{(a)}(\overline{\mathbf{B}} \cdot(\nabla \times \overline{\mathbf{B}})) \\
& +(\mathbf{e} \cdot \overline{\mathbf{B}})\left(\frac{1}{3} f_{4}^{(d)}(\overline{\mathbf{B}} \cdot \mathbf{G})+\frac{4 f_{9}^{(a)}}{(\varepsilon+1)}(\overline{\mathbf{B}} \cdot \mathbf{U})\right)-f_{4}^{(d)} \frac{(\mathbf{e} \cdot \nabla)}{6} \bar{B}^{2} \\
& \left.-\frac{4}{3} f_{1}^{(a)}(\mathbf{e} \cdot \overline{\mathbf{B}})(\mathbf{e} \cdot(\nabla \times \overline{\mathbf{B}}))-f_{3}^{(d)} \frac{(\mathbf{e} \cdot \nabla)}{6}(\mathbf{e} \cdot \overline{\mathbf{B}})^{2}-f_{4}^{(d)} \frac{(\mathbf{B} \cdot \nabla)}{3}(\mathbf{e} \cdot \overline{\mathbf{B}})\right\}
\end{aligned}
$$

where substitution $\left\langle u^{(0) 2}\right\rangle \ell_{c}^{-2} \rightarrow \tau_{c}^{-2}$ was used, and $\mathbf{e}=\boldsymbol{\Omega} /|\Omega|$. Here, we dropped the contributions due to shear because their effect to the mean electromotive force was computed only to the zero order terms about angular velocity. Furthermore, in (45) we kept only those contributions which could be the most interesting from the stellar dynamo applications standpoint. Note, for the equipartition case, $\varepsilon=1$, helicity evolution satisfies the simple equation:

$$
\frac{\partial h_{\mathcal{C}}}{\partial t}+\frac{1}{\tau_{h}} h_{\mathcal{C}}=-\frac{2(\mathcal{E} \cdot \overline{\mathbf{B}})}{\mu \rho \ell_{c}^{2}} .
$$

It is in accordance with equation for the magnetic helicity density obtained by Subramanian and Brandenburg (2005). As an example of application of (46) to the problem of the nonlinear saturation of alphaeffect, consider the $\alpha^{2}$ dynamo in the fast rotation limit. For the sake of simplicity we restrict ourselves only with the isotropic components of $\alpha$-effect and neglect the helicity loss due to $h_{\mathcal{C}} / \tau_{h}$. From (46) and (26) we get

$$
\frac{\partial h_{\mathcal{C}}}{\partial t}=\frac{\pi \beta^{2}}{4 \tau_{c}}\left(2\left\langle u^{(0) 2}\right\rangle \Omega^{*}(\mathbf{e} \cdot \mathbf{G})-h_{\mathcal{C}}\right)
$$

where we keep the contributions of order $\Omega^{*-1}$ for the current helicity, and drop the terms which are due to nonuniform LSMF. If $L$ is the typical spatial scale of the LSMF then the eq. (47) is justified when $L G \Omega^{*} \gg 1$ and $\mu \rho\left|h_{\mathcal{C}}\right| \gg|\overline{\mathbf{B}} \cdot(\nabla \times \overline{\mathbf{B}})|$. The point to note that in (47) we implicitly assume that $h_{\mathcal{C}}^{(0)} \equiv h_{\mathcal{C}}$. It is a shortcoming of the theory. However, this procedure is widely used in the literature (Kleeorin and Ruzmaikin, 1982; Vainshtein and Kitchatinov, 1983; Vishniac and Cho, 2001; Kleeorin et al., 2003; Brandenburg and Subramanian, 2004). With initial condition, $t=0, h_{\mathcal{C}}=0$, we write, similar to Vainshtein (1983), the solution of eq (47) as follows,

$$
h_{\mathcal{C}}=2 \Omega^{*}\left\langle u^{(0) 2}\right\rangle(\mathbf{e} \cdot \mathbf{G})\left(1-\exp \left(-\frac{\pi}{4 \tau_{c}} \int_{0}^{t} \beta^{2} d t\right)\right) .
$$

The given solution shows that under $t \rightarrow \infty$ we get $h_{\mathcal{C}} \rightarrow 2 \Omega^{*}\left\langle u^{(0) 2}\right\rangle(\mathbf{e} \cdot \mathbf{G}) \tau_{c}$. On this basis, and in taking into account (26), we can conclude that $\alpha$-effect will saturates exponentially under the increase of the LSMF strength. Furthermore, this conclusion was confirmed with numerical dynamo model which is considered by author in the separate paper (Pipin, 2007).

Next, we consider the equation for the current helicity evolution for the slow rotation limit. No restriction is applied to the strength of LSMF. The contribution of shear to the transport and generation part of equation is described with a quite bulky tensor expressions and we decide to restrict ourselves with terms which have either a finite limit under $\beta \rightarrow 0$ or the amplitude functions that are greater than 0.1 . We write the evolutionary equation for the current helicity as follows:

$$
\begin{aligned}
\frac{\partial h_{\mathcal{C}}}{\partial t}+\frac{1}{\tau_{h}} h_{\mathcal{C}} & =-\frac{2}{\mu \rho \ell_{c}^{2}}(\mathcal{E} \cdot \overline{\mathbf{B}})+\psi_{1} \frac{\bar{B}_{m} \bar{B}_{p}}{\bar{B}^{2}} \bar{V}_{p, m} h_{\mathcal{C}}+\left(\psi_{2} \mathbf{G}+\psi_{3} \mathbf{U}\right) \cdot \mathbf{W}\left\langle u^{(0) 2}\right\rangle \\
& +\frac{1}{\mu \rho} \nabla \cdot\left(\left[\psi_{5} \nabla \times \overline{\mathbf{B}}+\psi_{4}(\mathbf{U} \times \overline{\mathbf{B}})\right](\overline{\mathbf{B}} \cdot \overline{\mathbf{V}})+\psi_{6} \mathbf{W} \bar{B}^{2}\right)+(\varepsilon-1)\{\ldots\},
\end{aligned}
$$


$\Omega$

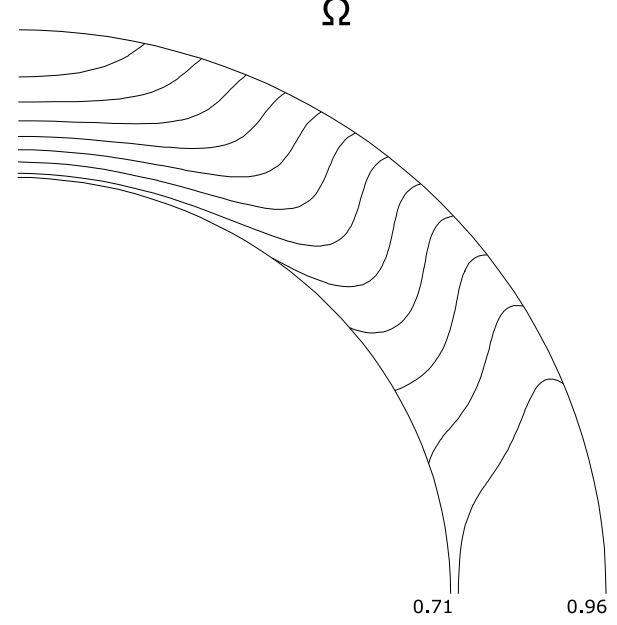

W

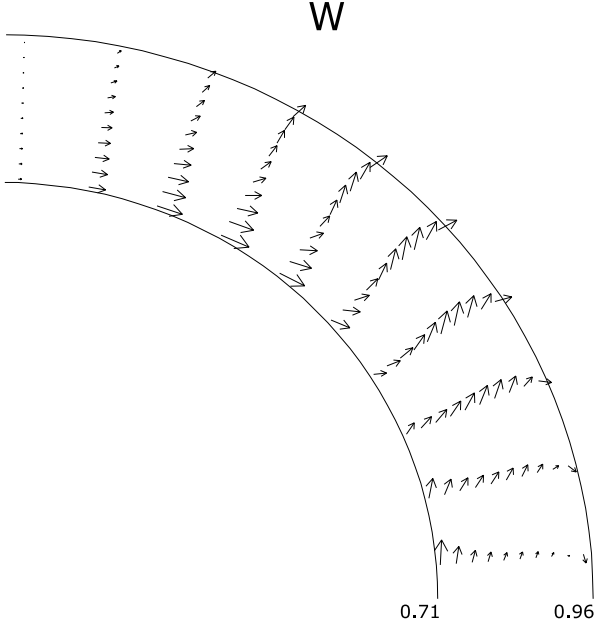

Figure 7: The isolines of the angular velocity distribution (left) and the corresponding vector field of the large-scale vorticity(right).

where $\mathbf{W}=\nabla \times \overline{\mathbf{V}}$. Quenching functions $\psi_{\{n\}}$ are given in Appendix A. Symbol $\{\ldots\}$ denotes those terms which are not important in the case $\varepsilon=1$. Taking the Taylor expansion of (49) for the case $\bar{B} \rightarrow 0$ (keeping $\bar{B}^{2}$ terms) we get

$$
\begin{aligned}
\frac{\partial h_{\mathcal{C}}}{\partial t}+\frac{1}{\tau_{h}} h_{\mathcal{C}} & =-\frac{2}{\mu \rho \ell_{c}^{2}}(\mathcal{E} \cdot \overline{\mathbf{B}})-\frac{4}{15} \frac{\bar{B}_{m} \bar{B}_{p}}{\mu \rho\left\langle u^{(0) 2}\right\rangle} V_{p, m} h_{\mathcal{C}}-\frac{(\mathbf{G} \cdot \mathbf{W})}{6}\left\langle u^{(0) 2}\right\rangle-\nabla \cdot \mathcal{F} \\
\mathcal{F} & =\left(\frac{1}{6}\left\langle u^{(0) 2}\right\rangle+\frac{2}{15} \frac{\bar{B}^{2}}{\mu \rho}\right) \mathbf{W}+\frac{2}{15 \mu \rho}([\nabla \times \overline{\mathbf{B}}-(\mathbf{U} \times \overline{\mathbf{B}})](\overline{\mathbf{B}} \cdot \overline{\mathbf{V}}))
\end{aligned}
$$

where we apply the equipartition condition, $\varepsilon=1$, as well. The direction of the helicity flux due to the first contribution in (51), $\mathcal{F}^{\mathbf{W}}=\left(\left\langle u^{(0) 2}\right\rangle / 6+2 \bar{B}^{2} /(15 \mu \rho)\right) \mathbf{W}$, depends on distribution of the large-scale vorticity solely. The second term depends on details of the dynamo action. To estimate the direction of the helicity transport due to $\mathcal{F}^{\mathbf{W}}$ on the Sun we compute the vector field of the large-scale vorticity $\mathbf{W}$. In the spherical coordinate system we have $\mathbf{W}=\sin \theta \mathbf{e}^{r} \partial \Omega / \partial \theta-r \sin \theta \mathbf{e}^{\theta} \partial \Omega / \partial r$, where $r, \theta$ are the radial distance and the polar angle, respectively. The distribution of the angular velocity is taken as an analytical fit given by Belvedere et al. (2000). It is shown at the left side Fig:7 The computed vector field of the large-scale vorticity is shown at the right side Fig 7

The given figure shows the possibility of the outward helicity flux from the dynamo region due to shear. Note that one component of the helicity flux $\mathcal{F}^{\mathbf{W}}$ is due to the small-scale dynamo, $\left\langle u^{(0) 2}\right\rangle \mathbf{W} / 6$, and another is due to the LSMF, $2 \bar{B}^{2} \mathbf{W} /(15 \mu \rho)$. Among two the contribution of the small-scale dynamo is likely to be dominated in the depth of convection zone. While the flux due to the LSMF may be important at near the surface level. The latter effect may produce the significant outward flux of helicity only with the open boundaries (Brandenburg and Subramanian, 2004; Subramanian and Brandenburg, 2005). At the near surface level the amplitude of the largescale vorticity, $|\mathbf{W}| \approx 4 \times 10^{-8} s^{-1} \approx 1.5 \times 10^{-5} d a y^{-1}$. The magnitude of the surface magnetic flux change during the solar cycle is about $10^{24} M x$ (Schrijver and Harvey, 1984). Then the magnitude of the helicity outflow from $2 \bar{B}^{2} \mathbf{W} /(15 \mu \rho)$ is about $10^{43} M x^{2} d a y^{-1}$. It is compatible with estimations given by DeVore (2000). We have estimated only one part of the helicity flux. The numerical dynamo model based on the given results would help to get a more definite conclusions about this subject. 


\section{Summary}

In the paper the mean electromotive force of turbulent flows and magnetic fields is computed analytically using the framework of mean-field dynamo theory and MTA (minimal $\tau$ approximation). There is an overlap in results obtained with SOCA and with MTA approximation. The two approximations give qualitatively the same results about nonlinear dependence of mean electromotive force on the strength of LSMF or on the Coriolis number. Also there is a difference between predictions of SOCA and MTA for mean-electromotive force expressions if the shear is taken into account. This difference can be explained, in part, by the crudeness of the given version of tau approximation. The deficient accuracy of calculations of shear contribution is due to an assumption about the scale-independent $\tau$. In whole, the accuracy of the theory presented in the paper is comparable with the mixing-length approximation. It has no the firm grounds and should be considered with caution.

Finally, I would like to focus on the new findings of the paper. In this study it is shown that the new interesting component of transport of LSMF appears due to joint contribution of current helicity and shear. The effect does not disappear in the strong LSMF limit, $\beta \gg 1$. It may be important near the base of solar CZ where the influence of rotation and shear on the turbulence is quite strong. Furthermore, the analysis, which we carried out for the current helicity evolution, suggests that the shear and rotation may redistribute the helicity in solar CZ amplifying it (in amplitude) at the near equatorial regions in agreement with observations. Beside, the effect of rotation and stratification on the $h_{\mathcal{C}}$ evolution is calculated explicitly. Basically, the equation for current helicity is obtained using the same approach as for the mean electromotive force and on the base of quantities which are explicitly gauge invariants. Therefore, we can expect that the dynamo model based on the above approach could be capable for meeting the requirements of both solar and stellar dynamo simulations.

Acknowledgments This work benefited from the research grants 05-02-16326 and 4741.2006.2. I thank sincerely K.-H. Rädler and A. Brandenburg for criticism and helpful suggestions. I acknowledge valuable discussion with Nathan Kleeorin and Igor Rogachevskii. Gratitude also goes to Kirill Kuzanyan for the critical reading manuscript. The large portion of calculations presented in the paper were done with help of Maxima computer algebra system (http://Maxima.sf.net). I owe sincere thanks to all developers of Maxima and personally to Victor Toth (Toth, 2005) for maintaining the Maxima's package for tensor calculations.

\section{References}

Belvedere, G., Kuzanyan, K. M., Sokoloff, D., A two-dimensional asymptotic solution for a dynamo wave in the light of the solar internal rotation. Mon. Not. R. Astron. Soc. 315, 778-790(2000).

Blackman, E. G., Brandenburg, A., Dynamic nonlinearity in large scale dynamos with shear. Astrophys. J. 579, 379-373(2002).

Blackman, E. G., Field, G. B., New dynamical mean-field dynamo theory and closure approach. Phys. Rev. Lett. $89,265007(2002)$.

Brandenburg, A., The inverse cascade and nonlinear alpha effect in simulations of isotropic helical hydromagnetic turbulence. Astrophys. J. 550, 824-840(2001).

Brandenburg, A., Subramanian, K., Strong mean field dynamos require supercritical helicity fluxes. Astron. Nachr. 325, 400-408(2004).

Brandenburg, A., Subramanian, K., 2005. Astrophysical magnetic fields and nonlinear dynamo theory. Phys. Rep. 417, 1-209.

DeVore, C. R., Magnetic helicity generation by solar differential rotation. Astrophys. J. 539, 944-953(2000). 
Field, G. B., Blackman, E. G., Dynamical quenching of the alpha ${ }^{2}$ dynamo. Astrophys. J. 572, 685-692(2002).

Frisch, U., Pouquet, A., Léorat, J., A., M., Possibility of an inverse cascade of magnetic helicity in magnetohydrodynamic turbulence. J. Fluid Mech. 68, 769-778(1975).

Kichatinov, L. L., Turbulent transport of magnetic fields in a highly conducting rotating fluid and the solar cycle. Astron. Astrophys. 243, 483-491(1991).

Kichatinov, L. L., Turbulent transport in rotating and magnetized fluids. R. Erdelyi et al(eds.), Turbulence, Waves and Instabilities in the Solar Plasma Kluwer, 81-96(2003).

Kichatinov, L. L., Pipin, V., Rüdiger, G., Turbulent viscosity, magnetic diffusivity, and heat conductivity under the influence of rotation and magnetic field. Astron. Nachr. 315, 157-170(1994).

Kichatinov, L. L., Rüdiger, G., Magnetic-field advection in inhomogeneous turbulence. Astron. Astrophys. 260, 494-498(1992).

Kleeorin, N., Kuzanyan, K., Moss, D., Rogachevskii, I., Sokoloff, D., H., Z., Magnetic helicity evolution during the solar activity cycle: Observations and dynamo theory. Astron. Astrophys. 409, 1097-1105(2003).

Kleeorin, N., Rogachevskii, I., Magnetic helicity tensor for an anisotropic turbulence. Phys. Rev.E $59,6724-6729(1999)$.

Kleeorin, N. I., Ruzmaikin, A. A., Dynamics of the average turbulent helicity in a magnetic field. Magnetohydrodynamics 18, 116-122(1982).

Krause, F., Rädler, K.-H., Mean-Field Magnetohydrodynamics and Dynamo Theory. Berlin: Akademie-Verlag(1980).

Moffatt, H. K., Magnetic Field Generation in Electrically Conducting Fluids. Cambridge, England: Cambridge University Press(1978).

Orszag, S., Analytical theories of turbulence. J. Fluid Mech. 41, 363(1970).

Parker, E. N., Cosmical magnetic fields: Their origin and their activity. Oxford: Clarendon $\operatorname{Press}(1979)$.

Pipin, V. V., The generation of the solar magnetic field in $\alpha \delta \Omega$ dynamo. Astronomy Rep. accepted (2007).

Pouquet, A., Frisch, U., Léorat, J., Strong MHD helical turbulence and the nonlinear dynamo effect. J. Fluid Mech. 68, 769-778(1975).

Rädler, K.-H., On the electrodynamics of turbulent fields under the influence of corilois forces. Monats. Dt. Akad. Wiss. 11, 194-201(1969).

Rädler, K.-H., Kleeorin, N., Rogachevskii, I., The mean electromotive force for mhd turbulence: the case of a weak mean magnetic field and slow rotation. Geophys. Astrophys. Fluid Dyn. 97, 249-269(2003).

Rädler, K.-H., Rheinhardt, M., Mean-field electrodynamics: Critical analysis of various analytical approaches to the meanelectromotive force. Geophys. Astrophys. Fluid Dyn. accepted, (astro$\mathrm{ph} / 0606267)(2006)$.

Rädler, K.-H., Stepanov, R., The mean electromotive force due to turbulence of a conducting fuild in the presence of mean flow. Phys. Rev. 73 (056311), 1-15(2006).

Roberts, P., Soward, A., A unified approach to mean field electrodynamics. Astron. Nachr. 296, 49-64(1975). 
Rogachevskii, I., Kleeorin, N., Electromotive force and large-scale magnetic dynamo in a turbulent flow with a mean shear. Phys. Rev.E 68 (036301), 1-12(2003).

Rogachevskii, I., Kleeorin, N., Effects of differential and uniform rotation on nonlinear electromotive force in a turbulent flow. arXiv:astro-ph/0407375v2(2004a).

Rogachevskii, I., Kleeorin, N., 2004b. Nonlinear theory of a "shear-current" effect and mean-field magnetic dynamos. Phys. Rev.E 70 (046310), 1-15(2004b).

Rogachevskii, I., Kleeorin, N., Shear-current effect in a turbulent convection with a large-scale shear. Phys. Rev.E (accepted)(2007).

Rüdiger, G., Kichatinov, L. L., Alpha-effect and alpha-quenching. Astron. Astrophys. 269, 581$588(1993)$.

Rüdiger, G., Kichatinov, L. L., Do mean-field dynamos in nonrotating turbulent shear-flows exist? Astron. Nachr. 327, 298-303(2006).

Schrijver, C., Harvey, K., The photospheric magnetic flux budget. Sol.Phys. 150, 1-10(1984).

Subramanian, K., Brandenburg, A., Nonlinear current helicity fluxes in turbulent dynamos and alpha quenching. Phys. Rev. Lett. 93, 205001(2004).

Subramanian, K., Brandenburg, A., Magnetic helicity density and its flux in weakly inhomogeneous turbulence, Astrophys.J., 648,L71-L74,astro-ph/0509392(2006).

Toth, V., Tensor manipulation in GPL Maxima, cs.SC/0503073(2005).

Vainshtein, S. I., Magnitnie polia v cosmose. Moscow, Nauka(1983).

Vainshtein, S. I., Kitchatinov, L. L., The macroscopic magnetohydrodynamics of inhomogeneously turbulent cosmic plasmas. Geophys. Astrophys. Fluid Dynam. 24, 273-298(1983).

Vishniac, E. T., Cho, J., Magnetic helicity conservation and astrophysical dynamos. Astrophys. J. 550, 752-760(2001). 


\section{Appendix A.}

This part of appendix gives the functions of the Coriolis number defining the dependence of the turbulent transport generation and diffusivities on the angular velocity.

$$
\begin{aligned}
f_{1}^{(a)} & =\frac{1}{4 \Omega^{* 2}}\left(\left(\Omega^{* 2}+3\right) \frac{\arctan \Omega^{*}}{\Omega^{*}}-3\right), \\
f_{2}^{(a)} & =\frac{1}{4 \Omega^{* 2}}\left(\left(\Omega^{* 2}+1\right) \frac{\arctan \Omega^{*}}{\Omega^{*}}-1\right), \\
f_{3}^{(a)} & =\frac{1}{4 \Omega^{* 2}}\left(\left((\varepsilon-1) \Omega^{* 2}+\varepsilon-3\right) \frac{\arctan \Omega^{*}}{\Omega^{*}}+3-\varepsilon\right), \\
f_{4}^{(a)} & =\frac{1}{6 \Omega^{* 3}}\left(3\left(\Omega^{* 4}+6 \varepsilon \Omega^{* 2}+10 \varepsilon-5\right) \frac{\arctan \Omega^{*}}{\Omega^{*}}-\left((8 \varepsilon+5) \Omega^{* 2}+30 \varepsilon-15\right)\right), \\
f_{5}^{(a)} & =\frac{1}{3 \Omega^{* 3}}\left(3\left(\Omega^{* 4}+3 \varepsilon \Omega^{* 2}+5(\varepsilon-1)\right) \frac{\arctan \Omega^{*}}{\Omega^{*}}-\left((4 \varepsilon+5) \Omega^{* 2}+15(\varepsilon-1)\right)\right), \\
f_{6}^{(a)} & =-\frac{1}{48 \Omega^{* 3}}\left(3\left((3 \varepsilon-11) \Omega^{* 2}+5 \varepsilon-21\right) \frac{\arctan \Omega^{*}}{\Omega^{*}}-\left(4(\varepsilon-3) \Omega^{* 2}+15 \varepsilon-63\right)\right), \\
f_{7}^{(a)} & =\frac{1}{48 \Omega^{* 3}}\left(3\left((5 \varepsilon+3) \Omega^{* 2}+11 \varepsilon+5\right) \frac{\arctan \Omega^{*}}{\Omega^{*}}-\left(4(\varepsilon+1) \Omega^{* 2}+33 \varepsilon+15\right)\right), \\
f_{8}^{(a)} & =-\frac{1}{12 \Omega^{* 3}}\left(3\left((3 \varepsilon+1) \Omega^{* 2}+4 \varepsilon-2\right) \frac{\arctan \Omega^{*}}{\Omega^{*}}-\left(5(\varepsilon+1) \Omega^{* 2}+12 \varepsilon-6\right)\right), \\
f_{9}^{(a)} & =\frac{(\varepsilon+1)}{4 \Omega^{*}}\left(\frac{\arctan \Omega^{*}}{\Omega^{*}}-1\right), \\
f_{10}^{(a)} & =-\frac{1}{3 \Omega^{* 3}}\left(3\left(\Omega^{* 2}+1\right)\left(\Omega^{* 2}+\varepsilon-1\right) \frac{\arctan \Omega^{*}}{\Omega^{*}}-\left((2 \varepsilon+1) \Omega^{* 2}+3 \varepsilon-3\right)\right), \\
f_{11}^{(a)} & =-\frac{1}{6 \Omega^{* 3}}\left(3\left(\Omega^{* 2}+1\right)\left(\Omega^{* 2}+2 \varepsilon-1\right) \frac{\arctan \Omega^{*}}{\Omega^{*}}-\left((4 \varepsilon+1) \Omega^{* 2}+6 \varepsilon-3\right)\right) .
\end{aligned}
$$

The dependence of turbulent diffusivities on the Coriolis number (eq. (28)) is given by

$$
\begin{aligned}
f_{1}^{(d)} & =\frac{1}{2 \Omega^{* 3}}\left((\varepsilon+1) \Omega^{* 2}+3 \varepsilon-\left((2 \varepsilon+1) \Omega^{* 2}+3 \varepsilon\right) \frac{\arctan \left(\Omega^{*}\right)}{\Omega^{*}}\right), \\
f_{2}^{(d)} & =\frac{1}{4 \Omega^{* 2}}\left(\left((\varepsilon-1) \Omega^{* 2}+3 \varepsilon+1\right) \frac{\arctan \left(\Omega^{*}\right)}{\Omega^{*}}-(3 \varepsilon+1)\right), \\
f_{3}^{(d)} & =\frac{1}{2 \Omega^{* 3}}\left(3\left(3 \Omega^{* 2}+5\right) \frac{\arctan \left(\Omega^{*}\right)}{\Omega^{*}}-\left(4 \Omega^{* 2}+15\right)\right), \\
f_{4}^{(d)} & =\frac{1}{2 \Omega^{* 3}}\left(\left(2 \Omega^{* 2}+3\right)-3\left(\Omega^{* 2}+1\right) \frac{\arctan \left(\Omega^{*}\right)}{\Omega^{*}}\right) .
\end{aligned}
$$

The magnetic quenching functions of the generation and transport effects in eq. (33) are 


$$
\begin{aligned}
& \varphi_{1}^{(a)}=\frac{1}{64 \beta^{2}}\left(\left(4(3 \varepsilon+1) \beta^{2}-17 \varepsilon+21\right) \frac{\arctan (2 \beta)}{2 \beta}+\frac{\left(4(11 \varepsilon-15) \beta^{2}+17 \varepsilon-21\right)}{\left(4 \beta^{2}+1\right)}\right), \\
& \varphi_{2}^{(a)}=\frac{(1-\varepsilon)}{8 \beta^{2}}\left(\frac{\arctan (2 \beta)}{2 \beta}-1\right) \text {, } \\
& \varphi_{3}^{(a)}=\frac{1}{3072 \beta^{4}}\left(\frac{8 \beta^{2}\left(2 \beta^{2}\left(16(45 \varepsilon+107) \beta^{2}-1731 \varepsilon+739\right)-2097 \varepsilon+97\right)-2295 \varepsilon-105}{\left(4 \beta^{2}+1\right)^{2}}\right. \\
& \text { - } \left.3\left(12 \beta^{2}\left(16(3 \varepsilon+5) \beta^{2}-41 \varepsilon+41\right)-765 \varepsilon-35\right) \frac{\arctan (2 \beta)}{2 \beta}\right), \\
& \varphi_{4}^{(a)}=\frac{1}{3072 \beta^{4}}\left(\frac{8 \beta^{2}\left(2 \beta^{2}\left(128(3 \varepsilon+1) \beta^{2}+807 \varepsilon-71\right)+555 \varepsilon-11\right)+459 \varepsilon+21}{\left(4 \beta^{2}+1\right)^{2}}\right. \\
& \left.-3\left(4(115 \varepsilon-19) \beta^{2}+153 \varepsilon+7\right) \frac{\arctan (2 \beta)}{2 \beta}\right) \text {, } \\
& \varphi_{5}^{(a)}=\frac{1}{3072 \beta^{4}}\left(3\left(4 \beta^{2}\left(48(3 \varepsilon+5) \beta^{2}-41 \varepsilon+41\right)-153 \varepsilon-7\right) \frac{\arctan (2 \beta)}{2 \beta}\right. \\
& \left.-\frac{4 \beta^{2}\left(16(9 \varepsilon+31) \beta^{2}-429 \varepsilon+109\right)-459 \varepsilon-21}{\left(4 \beta^{2}+1\right)}\right), \\
& \varphi_{6}^{(a)}=\frac{1}{3072 \beta^{4}}\left(3\left(4(163 \varepsilon-3) \beta^{2}-153 \varepsilon-7\right) \frac{\arctan (2 \beta)}{2 \beta}\right. \\
& \left.-\frac{4 \beta^{2}\left(64(21 \varepsilon+19) \beta^{2}+183 \varepsilon-23\right)-459 \varepsilon-21}{\left(4 \beta^{2}+1\right)}\right), \\
& \varphi_{7}^{(a)}=-\frac{1}{48 \beta^{4}}\left(3\left(2 \beta^{2}\left(4(\varepsilon+1) \beta^{2}-3 \varepsilon+3\right)-10 \varepsilon+5\right) \frac{\arctan (2 \beta)}{2 \beta}\right. \\
& \left.-\frac{\left(2 \beta^{2}\left(20(\varepsilon+1) \beta^{2}-49 \varepsilon+29\right)-30 \varepsilon+15\right)}{\left(4 \beta^{2}+1\right)}\right) \text {, }
\end{aligned}
$$

$$
\begin{aligned}
\varphi_{8}^{(a)} & =\frac{1}{48 \beta^{4}}\left(3\left(2 \varepsilon\left(2 \beta^{2}-1\right)+1\right) \frac{\arctan (2 \beta)}{2 \beta}-\right. \\
& \left.-\frac{\left(4 \beta^{2}\left(8(\varepsilon+1) \beta^{2}-\varepsilon+2\right)-6 \varepsilon+3\right)}{\left(4 \beta^{2}+1\right)}\right), \\
\varphi_{9}^{(a)} & =\frac{1}{48 \beta^{4}}\left(3\left(2 \beta^{2}\left(4(\varepsilon+1) \beta^{2}-\varepsilon+1\right)-2 \varepsilon+1\right) \frac{\arctan (2 \beta)}{2 \beta}-\left(2(\varepsilon+1) \beta^{2}-6 \varepsilon+3\right)\right), \\
\varphi_{10}^{(a)} & =\frac{1}{48 \beta^{4}}\left(\left(4(\varepsilon+1) \beta^{2}+6 \varepsilon-3\right)-3\left(2 \varepsilon\left(2 \beta^{2}+1\right)-1\right) \frac{\arctan (2 \beta)}{2 \beta}\right) .
\end{aligned}
$$

The nonlinear turbulent diffusion of the LSMF in (37) is expressed with help of the following functions 


$$
\begin{aligned}
& \varphi_{1}=\frac{(\varepsilon-1)}{16 \beta^{2}}\left(3 \frac{\arctan (2 \beta)}{\beta}-2 \frac{\left(8 \beta^{2}+3\right)}{\left(4 \beta^{2}+1\right)}\right), \\
& \varphi_{2}=\frac{(\varepsilon+1)}{32 \beta^{2}}\left(\left(4 \beta^{2}+3\right) \frac{\arctan (2 \beta)}{\beta}-3\right), \\
& \varphi_{3}=\frac{1}{8 \beta^{2}}\left(\frac{\arctan (2 \beta)}{\beta}-2\right) .
\end{aligned}
$$

The effect of slow rotation and nonuniform LSMF on the MEMF (eq. (38)) is expressed with help of the following functions

$$
\begin{aligned}
\varphi_{1}^{(w)} & =-\frac{1}{6144 \beta^{4}}\left(\frac{\left.4 \beta^{2}\left(4 \beta^{2}\left(4(51 \varepsilon-371) \beta^{2}+291 \varepsilon+29\right)-219 \varepsilon+1819\right)-315 \varepsilon+1275\right)}{\left(4 \beta^{2}+1\right)^{2}}\right. \\
& \left.+3\left(8 \beta^{2}\left(2(17 \varepsilon+47) \beta^{2}-51 \varepsilon+51\right)+105 \varepsilon-425\right) \frac{\arctan (2 \beta)}{2 \beta}\right) \\
\varphi_{2}^{(w)} & =-\frac{1}{6144 \beta^{4}}\left(3\left(8 \beta^{2}\left(2(37 \varepsilon+27) \beta^{2}+99 \varepsilon-99\right)+105 \varepsilon-425\right) \frac{\arctan (2 \beta)}{2 \beta}\right. \\
& \left.-\frac{4 \beta^{2}\left(4 \beta^{2}\left(4(273 \varepsilon+47) \beta^{2}+1269 \varepsilon-1589\right)+1119 \varepsilon-2719\right)+315 \varepsilon-1275}{\left(4 \beta^{2}+1\right)^{2}}\right) \\
\varphi_{3}^{(w)} & =\frac{1}{6144 \beta^{4}}\left(3\left(24 \beta^{2}\left(2(21 \varepsilon+43) \beta^{2}+125 \varepsilon-125\right)+735 \varepsilon-2975\right) \frac{\arctan (2 \beta)}{2 \beta}\right. \\
& \left.-\frac{4 \beta^{2}\left(4 \beta^{2}\left(4(1347 \varepsilon+125) \beta^{2}+5115 \varepsilon-8123\right)+5925 \varepsilon-17125\right)+2205 \varepsilon-8925}{\left(4 \beta^{2}+1\right)^{2}}\right), \\
\varphi_{4}^{(w)} & =\frac{1}{6144 \beta^{4}}\left(\frac{16 \beta^{2}\left((321 \varepsilon-1) \beta^{2}+165 \varepsilon-325\right)+315 \varepsilon-1275}{\left(4 \beta^{2}+1\right)}\right. \\
& \left.-3\left(8 \beta^{2}\left(2(21 \varepsilon+43) \beta^{2}+75 \varepsilon-75\right)+105 \varepsilon-425\right) \frac{\arctan (2 \beta)}{2 \beta}\right) \\
\varphi_{6}^{(w)} & =\varphi_{5}^{(w)}=\varphi_{4}^{(w)}, \\
\varphi_{7}^{(w)} & =\frac{1}{6144 \beta^{4}}\left(3\left(8 \beta^{2}\left(2(37 \varepsilon+27) \beta^{2}+33 \varepsilon-33\right)+21 \varepsilon-85\right) \frac{\arctan (2 \beta)}{2 \beta}\right. \\
& \left.-\frac{16 \beta^{2}\left((81 \varepsilon-17) \beta^{2}+60 \varepsilon-92\right)+63 \varepsilon-255}{\left(4 \beta^{2}+1\right)}\right) \\
\varphi_{8}^{(w)} & =\frac{1}{6144 \beta^{4}}\left(3\left(8 \beta^{2}\left(2(17 \varepsilon+47) \beta^{2}-17 \varepsilon+17\right)+21 \varepsilon-85\right) \frac{\arctan (2 \beta)}{2 \beta}\right. \\
& \left.\frac{16 \beta^{2}\left((51 \varepsilon-115) \beta^{2}+15 \varepsilon+17\right)-63 \varepsilon+255}{\left(4 \beta^{2}+1\right)}\right) \\
& \\
&
\end{aligned}
$$




$$
\begin{aligned}
\varphi_{1}^{(s)} & =\frac{1}{192 \beta^{4}}\left(3\left(-20 \varepsilon+3 \beta^{2}\left(-\varepsilon+4 \beta^{2}(\varepsilon-1)-7\right)+10\right) \frac{\arctan (2 \beta)}{2 \beta}\right. \\
& \left.-\frac{\left(\beta^{2}\left(8 \beta^{2}\left(2 \beta^{2}(23 \varepsilon-55)-67 \varepsilon-25\right)-409 \varepsilon+137\right)-60 \varepsilon+30\right)}{\left(4 \beta^{2}+1\right)^{2}}\right), \\
\varphi_{2}^{(s)} & =\frac{1}{192 \beta^{4}}\left(\frac{\left(\beta^{2}\left(4 \beta^{2}(7 \varepsilon-23)-35 \varepsilon-5\right)-12 \varepsilon+6\right)}{\left(4 \beta^{2}+1\right)}\right. \\
& \left.-3\left(-4 \varepsilon+\beta^{2}\left(-\varepsilon+12 \beta^{2}(\varepsilon-1)-7\right)+2\right) \frac{\arctan (2 \beta)}{2 \beta}\right), \\
\varphi_{3}^{(s)} & =\frac{(\varepsilon+1)}{16 \beta^{2}}\left(\left(4 \beta^{2}+3\right) \frac{\arctan (2 \beta)}{2 \beta}-3\right), \\
\varphi_{4}^{(s)} & =-\frac{(\varepsilon+1)}{8 \beta^{2}}\left(\frac{\arctan (2 \beta)}{2 \beta}-1\right), \\
\varphi_{5}^{(s)} & =\frac{1}{96 \beta^{4}}\left(8 \beta^{2}(\varepsilon+1)-6 \varepsilon+3-3\left(4 \beta^{2}+1-2 \varepsilon\right) \frac{\arctan (2 \beta)}{2 \beta}\right)
\end{aligned}
$$

The quenching functions of the current helicity effects obtained in the paper are

$$
\begin{aligned}
\varphi_{1}^{(h)} & =\frac{1}{4 \beta^{2}}\left(1-\frac{\arctan (2 \beta)}{2 \beta}\right), \\
\varphi_{2}^{(h)} & =\frac{1}{64 \beta^{2}}\left(3\left(4 \beta^{2}+5\right) \frac{\arctan (2 \beta)}{2 \beta}-\frac{5\left(4 \beta^{2}+3\right)}{\left(4 \beta^{2}+1\right)}\right), \\
\varphi_{3}^{(h)} & =-\frac{1}{192 \beta^{4}}\left(3\left(12 \beta^{4}-\beta^{2}-8\right) \frac{\arctan (2 \beta)}{2 \beta}+\frac{\left(4 \beta^{4}+67 \beta^{2}+24\right)}{\left(4 \beta^{2}+1\right)}\right), \\
\varphi_{4}^{(h)} & =\frac{1}{96 \beta^{4}}\left(3\left(12 \beta^{4}-3 \beta^{2}-20\right) \frac{\arctan (2 \beta)}{2 \beta}-\frac{\left(368 \beta^{6}-536 \beta^{4}-409 \beta^{2}-60\right)}{\left(4 \beta^{2}+1\right)^{2}}\right) .
\end{aligned}
$$


The magnetic quenching functions for the shear-current effects are

$$
\begin{aligned}
& \varphi_{1}^{(V)}=\frac{1}{(8 \beta)^{4}}\left(\left(16 \beta^{2}\left(\beta^{2}(125 \varepsilon+43)-25 \varepsilon-3\right)-\varepsilon-87\right) \frac{\arctan (2 \beta)}{2 \beta}\right. \\
& \left.-\frac{\left(8 \beta^{2}\left(2 \beta^{2}\left(128 \beta^{2}(3 \varepsilon+13)-19 \varepsilon-709\right)-755 \varepsilon-525\right)-15 \varepsilon-1305\right)}{15\left(4 \beta^{2}+1\right)}\right), \\
& \varphi_{2}^{(V)}=\frac{1}{2(8 \beta)^{4}}\left(\left(8 \beta^{2}\left(2 \beta^{2}(151 \varepsilon-15)-15 \varepsilon-57\right)-125 \varepsilon+165\right) \frac{\arctan (2 \beta)}{2 \beta}\right. \\
& \left.-\frac{\left(16 \beta^{2}\left(\beta^{2}(443 \varepsilon-339)-85 \varepsilon-3\right)-375 \varepsilon+495\right)}{3\left(4 \beta^{2}+1\right)}\right), \\
& \varphi_{3}^{(V)}=-\frac{1}{2(8 \beta)^{4}}\left(\left(8 \beta^{2}\left(2 \beta^{2}(247 \varepsilon-367)+193 \varepsilon-393\right)-125 \varepsilon+165\right) \frac{\arctan (2 \beta)}{2 \beta}\right. \\
& \left.+\frac{\left(16 \beta^{2}\left(\beta^{2}\left(512 \beta^{2}(\varepsilon+3)-1563 \varepsilon+2739\right)-227 \varepsilon+507\right)+375 \varepsilon-495\right)}{3\left(4 \beta^{2}+1\right)}\right), \\
& \varphi_{4}^{(V)}=\frac{1}{2(4 \beta)^{4}}\left(\left(\beta^{2}\left(4 \beta^{2}(17 \varepsilon-5)+5 \varepsilon+51\right)+10 \varepsilon-23\right) \frac{\arctan (2 \beta)}{2 \beta}\right. \\
& \left.+\frac{\left(\beta^{2}\left(4 \beta^{2}\left(64 \beta^{2}(\varepsilon+15)-609 \varepsilon-235\right)-475 \varepsilon+155\right)-150 \varepsilon+345\right)}{15\left(4 \beta^{2}+1\right)}\right), \\
& \varphi_{5}^{(V)}=-\frac{1}{2(4 \beta)^{4}}\left(\left(8 \beta^{2}\left(\left(2 \beta^{2}+5\right) \varepsilon-6\right)-7 \varepsilon+4\right) \frac{\arctan (2 \beta)}{2 \beta}\right. \\
& \left.+\frac{\left(16 \beta^{2}\left(\beta^{2}((8 \beta-7)(8 \beta+7) \varepsilon+80)-20 \varepsilon+35\right)+105 \varepsilon-60\right)}{15\left(4 \beta^{2}+1\right)}\right), \\
& \varphi_{6}^{(V)}=-\frac{1}{2(4 \beta)^{4}}\left(\left(8 \beta^{2}\left(2 \beta^{2}(3 \varepsilon-4)+7 \varepsilon+10\right)+35 \varepsilon-40\right) \frac{\arctan (2 \beta)}{2 \beta}\right. \\
& \left.-\frac{\left(16 \beta^{2}\left(\beta^{2}\left(64 \beta^{2}(\varepsilon+1)+23 \varepsilon+44\right)+28 \varepsilon-5\right)+105 \varepsilon-120\right)}{3\left(4 \beta^{2}+1\right)}\right), \\
& \varphi_{7}^{(V)}=\frac{1}{(4 \beta)^{2}}\left(\left(4 \beta^{2}(8 \varepsilon-7)-4 \varepsilon-41\right) \frac{\arctan (2 \beta)}{2 \beta}\right. \\
& \left.-\frac{\left(8 \beta^{2}\left(2 \beta^{2}\left(16 \beta^{2}(3 \varepsilon+1)+48 \varepsilon-67\right)+2 \varepsilon-113\right)-12 \varepsilon-123\right)}{3\left(4 \beta^{2}+1\right)^{2}}\right), \\
& \varphi_{8}^{(V)}=-\frac{1}{128 \beta^{4}}\left(\left(8 \beta^{2}\left(2 \beta^{2}(4 \varepsilon+5)+3\right)+5\right) \frac{\arctan (2 \beta)}{2 \beta}-\frac{\left(16 \beta^{2}\left(\beta^{2}(12 \varepsilon+25)+7\right)+15\right)}{3\left(4 \beta^{2}+1\right)}\right), \\
& \varphi_{9}^{(V)}=-\frac{1}{(8 \beta)^{4}}\left(\left(8 \beta^{2}\left(2 \beta^{2}(9 \varepsilon+47)-11 \varepsilon+19\right)+25 \varepsilon-65\right) \frac{\arctan (2 \beta)}{2 \beta}\right. \\
& \text { - } \left.\frac{1}{15}\left(4 \beta^{2}\left(256 \beta^{2}(\varepsilon+1)-455 \varepsilon+895\right)+375 \varepsilon-975\right)\right), \\
& \varphi_{10}^{(V)}=-\frac{1}{128 \beta^{2}}\left(\left(4 \beta^{2}(3 \varepsilon-11)+13 \varepsilon-21\right) \frac{\arctan (2 \beta)}{2 \beta}+\frac{1}{3}\left(16 \beta^{2}(\varepsilon+3)-39 \varepsilon+63\right)\right) \text {, } \\
& \varphi_{11}^{(V)}=-\frac{(\varepsilon+1)}{48 \beta^{2}}\left(3\left(4 \beta^{2}+1\right) \frac{\arctan (2 \beta)}{2 \beta}-8 \beta^{2}-3\right) \text {. }
\end{aligned}
$$


The magnetic quenching functions for the current helicity evolution equation:

$$
\begin{aligned}
\psi_{1} & =-\frac{\varphi_{3}^{(s)}}{(\varepsilon+1)}, \\
\psi_{2} & =-\frac{1}{768 \beta^{2}}\left(3\left(12(\varepsilon-1) \beta^{2}-21 \varepsilon+5\right) \frac{\arctan (2 \beta)}{2 \beta}\right. \\
& \left.+\frac{\left(4 \beta^{2}\left(32 \beta^{2}(\varepsilon+1)+65 \varepsilon-1\right)+63 \varepsilon-15\right)}{\left(4 \beta^{2}+1\right)}\right), \\
\psi_{3} & =\frac{1}{48 \beta^{2}}\left(3(\varepsilon-1) \frac{\arctan (2 \beta)}{2 \beta}-\left(4 \beta^{2}(\varepsilon+1)+3 \varepsilon-3\right)\right), \\
\psi_{4} & =\frac{1}{192 \beta^{4}}\left(\frac{\left.\left(4 \beta^{2}\left(15 \varepsilon+32 \beta^{2}+5\right)+9 \varepsilon+3\right)\right)}{\left(4 \beta^{2}+1\right)}-3(3 \varepsilon+1)\left(4 \beta^{2}+1\right) \frac{\arctan (2 \beta)}{2 \beta}\right), \\
\psi_{5} & =\frac{1}{8(2 \beta)^{4}}\left(\left(11 \varepsilon+4 \beta^{2}\left(-\varepsilon+8 \beta^{2}(\varepsilon-1)+5\right)-7\right) \frac{\arctan (2 \beta)}{2 \beta}\right. \\
& \left.-\frac{1}{15}\left(8 \beta^{2}\left(8 \beta^{2}(\varepsilon+1)-35 \varepsilon+55\right)+165 \varepsilon-105\right)\right), \\
\psi_{6} & =\frac{\left(4 \beta^{2}+4-3 \varepsilon\right)}{48 \beta^{4}}\left(3 \frac{\arctan (2 \beta)}{2 \beta}-\frac{\left.\left(8 \beta^{2}+3\right)\right)}{\left(4 \beta^{2}+1\right)}\right)
\end{aligned}
$$

\section{Appendix B. Comparison with some of results given in the paper by Rädler \& Stepanov (2006).}

This part of the article contains the comparison some of our results with those from RS06. We apply the mixing-length (MLT) approximation to expressions obtained in RS06. In this procedure we replace the spectrum of turbulent fields by the single-scaled function of the form $\delta\left(k-\ell_{r}^{-1}\right) \delta(\omega)$, where $\ell_{c}$ is the correlation length of the turbulence and put $\eta k^{2}=\nu k^{2}=\tau_{c}^{-1}$, Kichatinov, 1991).

The effect of stratification and shear. The structure of the electromotive force obtained by RS06 can be reproduced if we decompose the gradient of the large-scale flow $\bar{V}_{i, j}$ into symmetric and antisymmetric parts via

$$
\bar{V}_{i, j}=D_{i j}-\frac{1}{2} \varepsilon_{i j n} W_{n}
$$

where $W_{i}=\varepsilon_{i n m} \bar{V}_{m, n}$ is the large-scale vorticity and $D_{i j}=\left(\bar{V}_{i, j}+\bar{V}_{j, i}\right) / 2$ is the rate of strain tensor. After substitution (52) to (27) we obtain

$$
\begin{aligned}
\mathcal{E}_{i}^{(s)} & =\left(\varepsilon_{i n m} U_{k} \bar{B}_{n} D_{m k} A_{4}-\frac{A_{4}}{2}(\mathbf{W} \cdot \overline{\mathbf{B}}) U_{i}+(\mathbf{U} \cdot \overline{\mathbf{B}})\left(A_{3}-\frac{A_{2}}{2}+\frac{A_{1}}{2}+\frac{A_{4}}{2}\right) W_{i}\right. \\
& \left.+\varepsilon_{i n m} \bar{B}_{k} D_{n k} U_{m}\left(A_{2}+A_{1}\right)+(\mathbf{W} \cdot \mathbf{U}) \bar{B}_{i}\left(\frac{A_{2}}{2}-\frac{A_{1}}{2}\right)\right)\left\langle u^{(0) 2}\right\rangle . \\
& +\tau_{c}^{2} \frac{h_{\mathcal{C}}^{(0)}}{6}\left(\frac{5}{2}(\mathbf{W} \times \overline{\mathbf{B}})_{i}-\frac{23}{5} D_{i k} \bar{B}_{k}\right) .
\end{aligned}
$$


Using (53) we find

$$
\begin{aligned}
\tau_{c}^{-2} \tilde{\alpha}_{1}^{(W)} & =\frac{1}{2} \tau_{c}^{-2}\left(A_{1}-A_{2}\right)=0 \\
\tau_{c}^{-2} \tilde{\alpha}_{2}^{(W)} & =-\frac{1}{2} \tau_{c}^{-2}\left(A_{3}+\frac{1}{2}\left(A_{1}-A_{2}\right)\right)=-\frac{1}{12} \\
\tau_{c}^{-2} \tilde{\gamma}^{(W)} & =-\frac{1}{2} \tau_{c}^{-2}\left(A_{3}+A_{4}+\frac{1}{2}\left(A_{1}-A_{2}\right)\right)=0 \\
\tau_{c}^{-2} \tilde{\gamma}^{(D)} & =\frac{1}{2} \tau_{c}^{-2}\left(3 A_{4}-A_{1}-A_{2}\right)=-\frac{11}{60} \\
\tau_{c}^{-2} \tilde{\alpha}^{(D)} & =\frac{1}{2} \tau_{c}^{-2}\left(A_{4}-A_{1}-A_{2}\right)=-\frac{1}{60}
\end{aligned}
$$

where we put $\varepsilon=0$. After applying the MLT to results obtained in RS06 we find $\tau_{c}^{-2} \widetilde{\alpha}_{1}^{(W)}=$ $19 / 120, \tau_{c}^{-2} \widetilde{\alpha}_{2}^{(W)}=-7 / 240, \tau_{c}^{-2} \widetilde{\gamma}^{(W)}=-1 / 48, \tau_{c}^{-2} \widetilde{\gamma}^{(D)}=-39 / 120, \tau_{c}^{-2} \widetilde{\alpha}^{(D)}=-21 / 120$

The effect of nonuniform LSMF and shear. For the shear-current effect, after substitution of (52) to (30) we arrive to the following representation of $\mathcal{E}_{i}^{(V)}$,

$$
\begin{aligned}
\mathcal{E}_{i}^{(V)} & =\left\{\frac{C_{3}-C_{4}}{2}(\mathbf{W} \cdot \nabla) \bar{B}_{i}+\frac{C_{1}-C_{2}}{2} \nabla_{i}(\mathbf{W} \cdot \overline{\mathbf{B}})\right\}\left\langle u^{(0) 2}\right\rangle \\
& +\varepsilon_{i n m}\left\{\left(C_{1}+C_{2}\right) \bar{B}_{n, l}+\left(C_{3}+C_{4}\right) \bar{B}_{l, n}\right\} D_{m l}\left\langle u^{(0) 2}\right\rangle .
\end{aligned}
$$

Using this formula we obtain

$$
\begin{aligned}
\tau_{c}^{-2} \tilde{\delta}^{(W)} & =\frac{1}{4} \tau_{c}^{-2}\left(C_{3}-C_{4}-C_{1}+C_{2}\right)=\frac{1}{12} \\
\tau_{c}^{-2} \tilde{\kappa}^{(W)} & =\frac{1}{2} \tau_{c}^{-2}\left(C_{4}+C_{2}-C_{1}-C_{3}\right)=-\frac{4}{15} \\
\tau_{c}^{-2} \tilde{\kappa}^{(D)} & =-\frac{1}{2} \tau_{c}^{-2}\left(C_{1}+C_{2}+C_{3}+C_{4}\right)=\frac{3}{10} \\
\tau_{c}^{-2} \tilde{\beta}^{(D)} & =-\frac{1}{2} \tau_{c}^{-2}\left(C_{1}+C_{2}-C_{3}-C_{4}\right)=0
\end{aligned}
$$

where we put $\varepsilon=0$ in $C_{1-4}$. After applying the MLT to results in RS06 we find $\tau_{c}^{-2} \tilde{\delta}^{(W)}=1 / 12$, $\tau_{c}^{-2} \tilde{\kappa}^{(W)}=-1 / 30, \tau_{c}^{-2} \tilde{\kappa}^{(D)}=13 / 30$ and $\tau_{c}^{-2} \tilde{\beta}^{(D)}=7 / 60$. 